Illinois State University

ISU ReD: Research and eData

Theses and Dissertations

$7-29-2021$

\title{
A Multidimensional Analysis of Security Priming and Its Effect on Racial Essentialism and the Perception of Race
}

Marjorie Kate Hart

Illinois State University, mkatehart@comcast.net

Follow this and additional works at: https://ir.library.illinoisstate.edu/etd

\section{Recommended Citation}

Hart, Marjorie Kate, "A Multidimensional Analysis of Security Priming and Its Effect on Racial Essentialism and the Perception of Race" (2021). Theses and Dissertations. 1490.

https://ir.library.illinoisstate.edu/etd/1490

This Thesis is brought to you for free and open access by ISU ReD: Research and eData. It has been accepted for inclusion in Theses and Dissertations by an authorized administrator of ISU ReD: Research and eData. For more information, please contact ISUReD@ilstu.edu. 


\section{A MULTIDIMENSIONAL ANALYSIS OF SECURITY PRIMING AND ITS EFFECT ON RACIAL ESSENTIALISM AND THE PERCEPTION OF RACE}

\section{MARJORIE KATE HART}

\section{Pages}

Investigations into the perceptual impacts of priming a sense of attachment security have found that it improves emotional processing of attachment-related threat stimuli (Bai et al., 2019; Tang et al., 2017); however, no connections have been drawn for how those perceptual impacts effect the perception of ingroup and outgroup faces and whether there is potential for heightened attachment security to positively impact the way we perceive outgroup faces. Through multidimensional scaling (nMDS), I investigated how security priming influenced the other-race effect (the tendency to have increased processing only for same-race faces) and hypodescent (categorizing an ambiguous-race face as the minority race), while considering racial essentialism (rigid and biologically-based conceptions of race) as a potential moderator. One-hundred fifty participants (70\% White) were explicitly and subliminally primed in separate online sessions, then their implicit methods of categorization for Black, White, and racially ambiguous faces were gauged through 200 trials of similarity ratings. An analysis of the dimensions produced from the nMDS revealed that for all participants, regardless of ethnicity, the otherrace effect was pervasive for Black faces and participants relied twice as strongly on a race dimension to make category judgements. Neither secure attachment priming nor racial essentialism impacted implicit perceptual structures directly, and heightened security did not reduce hypodescent of ambiguous-race faces or racial essentialism. Higher racial essentialism predicted less individuation in the perceived race of Black faces, but it did not predict hypodescent of ambiguous-race faces. Supplementary analyses revealed the need for additional research into the interactions between security priming, ethnicity, and ingroup identification, as security priming tended to increase hypodescent in non-White participants and decrease individuation for those high in White ingroup identification. In conclusion, the repeated 
attachment security prime in this study failed to mitigate the processing biases of outgroup-race faces, and additional testing is required before considering implementing security priming in scenarios where outgroup-race face processing is crucial, such as in eye-witness identification duties.

KEYWORDS: secure attachment, other-race effect, cross-race effect, intergroup, racial essentialism, MDS 


\title{
A MULTIDIMENSIONAL ANALYSIS OF SECURITY PRIMING AND ITS EFFECT ON RACIAL ESSENTIALISM AND THE PERCEPTION OF RACE
}

\author{
MARJORIE KATE HART
}

A Thesis Submitted in Partial

Fulfillment of the Requirements for the Degree of

\section{MASTER OF SCIENCE}

Department of Psychology

\section{ILLINOIS STATE UNIVERSITY}

2021 
(C) 2021 Marjorie Kate Hart 


\title{
A MULTIDIMENSIONAL ANALYSIS OF SECURITY PRIMING AND ITS EFFECT ON RACIAL ESSENTIALISM AND THE PERCEPTION OF RACE
}

\author{
MARJORIE KATE HART
}

COMMITTEE MEMBERS:

Suejung Han, Co-chair

Matthew Hesson-McInnis, Co-chair 


\section{ACKNOWLEDGMENTS}

I thank both my advisors, S. Han for her invaluable editorial comments to this manuscript, and $\mathrm{M}$. Hesson-McInnis for providing the methodological inspiration and guidance, as well as the funding for this research. I also thank K. Pauker for providing the face stimuli, T. Critchfield for his editorial comments, and J. K. Golla for his constant support and for assisting in the creation of the figures.

M. K. H. 


\section{CONTENTS}

\section{Page}

ACKNOWLEDGMENTS

CONTENTS

TABLES

FIGURES

CHAPTER I: INTRODUCTION 1

CHAPTER II: LITERATURE REVIEW 3

Attachment System, Functioning, \& Styles 3

$\begin{array}{ll}\text { Secure Attachment Priming Effect } & 4\end{array}$

$\begin{array}{ll}\text { Face Perception Processing Biases } & 6\end{array}$

$\begin{array}{ll}\text { Racial Essentialism as a Moderator } & 9\end{array}$

$\begin{array}{ll}\text { Security Priming's Impact on Processing Biases } & 11\end{array}$

Using MDS for an Individual Differences Model $\quad 12$

Purpose of the Present Study 13

$\begin{array}{ll}\text { Hypotheses } & 14\end{array}$

CHAPTER III: METHODOLOGY AND RESULTS 16

$\begin{array}{ll}\text { Participants and Recruitment } & 16\end{array}$

$\begin{array}{ll}\text { Measures } & 17\end{array}$

$\begin{array}{ll}\text { Attachment } & 17\end{array}$

$\begin{array}{ll}\text { Racial Essentialism } & 18\end{array}$

$\begin{array}{ll}\text { Felt Security } & 18\end{array}$

$\begin{array}{ll}\text { Perceived Race } & 19\end{array}$

$\begin{array}{ll}\text { Ingroup Identification } & 19\end{array}$

$\begin{array}{ll}\text { Demographic Questionnaire } & 19\end{array}$ 
$\begin{array}{ll}\text { Materials } & 20\end{array}$

$\begin{array}{ll}\text { Explicit Priming } & 20\end{array}$

$\begin{array}{ll}\text { Implicit Priming Training Task } & 20\end{array}$

$\begin{array}{ll}\text { Face Stimuli } & 21\end{array}$

$\begin{array}{ll}\text { Procedure } & 22\end{array}$

$\begin{array}{ll}\text { Hypotheses } & 24\end{array}$

CHAPTER IV: RESULTS 26

$\begin{array}{ll}\text { Data Treatment } & 26\end{array}$

Preliminary Analyses and Hypotheses Testing 26

Hypothesis 1: External Validity of Racial Essentialism and Perceived Race Scale 27

Race as a Defining Dimension in Facial Perception: nMDS Analyses 27

Hypothesis 2: Common Race Dimension in All Solutions 28

Hypothesis 3a.1 - 3a.2: Security’s Impact on Perceptual Structures 29

Individual Differences Scaling for All Participants 31

Security Priming Effect Testing: MANOVAs 32

Factors Impacting the Prime Effect 34

Hypothesis 3a.3: Security Effects on Dimension Weights and Similarity Ratings 34

Hypothesis 4: Change in Racial Essentialism 35

Hypothesis 5: Prime, Racial Essentialism, and Perceived Race 35

Supplementary Analyses: A Moderating Role of Ingroup Identification and Ethnicity 37

White Ingroup Identification and Ethnicity Moderating Cross-Race Effect 38

Ethnicity Moderating Hypothesis 5: Effects of Secure Prime on Perceived Race 44

$\begin{array}{ll}\text { CHAPTER V: DISCUSSION } & 50\end{array}$

$\begin{array}{ll}\text { Interpretation of nMDS Dimensions } & 50\end{array}$

Racial Essentialism and Perceptions of Race $\quad 53$ 
Conclusions 


\section{TABLES}

Table

Page

1. Crosstabulation of Average Euclidean Distances Between Face Types by Prime Group 30

2. Cell Sizes of Racial-Ethnic Identities by Prime Group, Overall and Included in Analyses

3. Average Ratings of Perceived Race for Black, Ambiguous, and White Faces

4. Cell Sizes of White/Non-White Status by Prime Group

5. Between-Subjects Effects on Dimension Weights and Face Similarity Ratings

6. Ambiguous Similarity Ratings Predicted by White Ingroup Identification, Prime \& Felt Security

7. Multivariate Effects on the Variation in Same-Race Similarity Ratings

8. Multivariate Effects on Perceived Race Ratings and Variation Within Same-Race Faces

9. Between-Subjects Effects on Perceived Race and Variation in Race Ratings 


\section{FIGURES}

Figure $\quad$ Page

1. Example Face Stimuli 22

2. Comparison of Faces Distributed on Dimension 1 and the Perceived Race Scale 30

3. Four-Dimensional Individual Differences Model Generated with All Participant Matrices 32

4. Interaction of Prime Group and White/Non-White Status Across the Four Dimensions 42

5. White Ingroup and Similarity/Variation in Ambiguous Face Pair Ratings for White Participants 44

6. Black Faces: Prime Group and White/Non-White Status on Perceived Race Ratings 46

7. Ambiguous Faces : Prime Group and White/Non-White Status on Variation in Race Ratings 49 


\section{CHAPTER I: INTRODUCTION}

There is a continued rise of racial tensions in the United States as more cases of extrajudicial violence against and killings of Black people by law enforcement are highlighted in the media. These cases epitomize the larger and longstanding systemic inequity in the treatment of minorities by the justice system, where a recent study in Milwaukee County, Wyoming found that Black men, especially younger men, are significantly more likely to get sentenced to jail rather than probation compared to white men (Freiburger \& Sheeran, 2020); In a survey from 1990 to 2013, Black people were "3.6 times more likely to be incarcerated in local jails nationally than white people" (Subramanian et al., 2018, p. 22). There is even bias present in the simple process of eye-witness identification of suspects, where a phenomenon known as the other- or cross-race effect contributes to the misidentification of Black suspects by nonBlack witnesses (Dodson \& Dobolyi, 2016), leading to a disproportionate number of innocent Black citizens incarcerated (Reinitz, 2018).

All these scenarios motivate investigation into what factors can influence these critical decision points, when non-Black eyewitnesses, prosecutors, and law enforcement officers must rely on their own perceptions and judgements in the prosecution of Black individuals. The danger of the bias present in these scenarios is that the processes contributing to it are complex and likely subconscious in a way that is difficult to self-identify and prevent. For the present study, I identified one of the common mechanisms in these scenarios as the initial perception of race. The first motivation of this study was the question of how feelings of security influence the perception of others and how those feelings influence the perception and categorization of outgroup races. At what point do we perceive a face as being an outgroup race? Do feelings of security influence our racial categorizations? The scope of this study limits the ability to investigate how these perceptual processes then contribute to perceived threat, however, it is important to understand the basic perceptual processes and establish whether they are manipulable to provide a solid grounding from which to investigate behavioral outcomes in future studies.

I investigated factors contributing to the cross-race effect, where we tend to think that outgrouprace members look more alike and have more difficulty distinguishing them. Additionally, I measured 
racial essentialism - the belief that race is an innate, unchanging biological fact - as it is known to interact with racial categorization and predict hypodescent (assigning a face to its minority race by default). The ultimate goal of this study was to establish whether security priming can be a useful intervention for offsetting perceptual biases of outgroup-race members that would eventually lead to escalation and outgroup harm. Evidence of this effect would greatly enhance the utility of the security prime, indicating its potential as a non-intrusive intervention for threat-signal reduction and ultimately outgroup harm reduction.

There is already evidence for security priming being an effective intervention to increase positive affect, lower stigmatization of outgroup members, and improve emotional regulatory functioning when perceiving threatening stimuli (Rowe, Gold, \& Carnelley, 2020). There have also been investigations into the perceptual impacts of a security prime when viewing attachment-related threat stimuli (e.g. Bai et al., 2019), however no connections have been drawn to investigate how those perceptual impacts may be affecting the perception of ingroup and outgroup members.

To make the connection between security priming and the perception of outgroup members, I repeatedly primed participants with a secure attachment schema, then asked them to rate the similarity of White, Black, and Ambiguous faces (composed from Black and White parent faces), and afterwards rate the extent to which all faces appeared Black or White. By using multidimensional scaling methods to analyze these similarity ratings, I investigated the factors contributing to the cross-race effect and racial categorization, security priming's influence on those factors, and any interactions with racial essentialism and ingroup identification between the security primes, the cross-race effect, and racial categorization. 


\section{CHAPTER II: LITERATURE REVIEW}

In this next chapter I introduce the theory of the attachment system, affect regulation and the consequent development of a secure attachment prime. I then argue that the current literature regarding attachment security priming has yet to investigate security priming's impact on perceptual biases stemming from ingroup/outgroup and race perception. I identify the cross-race effect and racial categorization as testable manifestations of ingroup/outgroup bias in face processing, then address the potential for racial essentialism to act as a moderator for both. Next, I present evidence supporting the potential for a security prime to impact face processing and the resulting categorization of those faces. Lastly, I define multidimensional scaling as an excellent method for exploring the potential impacts of a security prime on face processing and categorization, and I introduce the purpose of the current study along with the hypotheses.

\section{Attachment System, Functioning, \& Styles}

The attachment system plays an important role in affect regulation, originating in infancy and developing over the lifespan (Bowlby, 1982). For infants and toddlers, whether they feel their caregiver functions as a secure base determines if that caregiver is sought out to attenuate distress, a process called proximity-seeking. Proximity-seeking persists in adult attachment styles, though it manifests differently in adulthood. Attachment figures are no longer always physically available, but proximity-seeking is accomplished via an internal working model that has been developed over the lifespan after having reinforcing interactions with attachment figure(s) (Shaver \& Mikulincer, 2002). This internal working model regulates emotions in attachment contexts (i.e. romantic relationships, family and close friend relationships), and can be activated merely by seeing attachment-related words like abandonment, love, or security (e.g. Mikulincer \& Shaver, 2001). This internal working model of attachment, or attachment style, also impacts the nature of our relationships as it maintains the representations of oneself, others, and the relation of oneself to others (Mikulincer, Shaver, \& Pereg, 2003).

Though it is common to talk of attachment styles as categorical traits, attachment style is best understood as a combination of two dimensions: anxiety and avoidance. The four-category model of 
attachment (secure, preoccupied, avoidant-fearful and avoidant-dismissive) has proved to be less robust in capturing the variance that the two-dimensional model captures. Low levels in both anxiety and avoidance characterize the secure attachment style, which, if in a situation of attachment-related threat, employs the primary attachment regulation strategies that involve increased cognitive accessibility of an attachment figure akin to proximity-seeking (Mikulincer et al., 2002). Primary attachment regulation strategies allow these securely attached individuals to naturally downregulate negative affective responses. In contrast, those with insecure attachment styles resort to secondary attachment strategies, hyperactivation and deactivation, which are less adaptive forms of affect regulation.

\section{Secure Attachment Priming Effect}

Because of the improved outcomes in a variety of domains for those with secure attachment in comparison to the insecure attachment styles, an intervention technique that primed a secure attachment schema was developed in hopes of improving outcomes despite dispositional attachment style

(Mikulincer \& Shaver, 2001). Secure attachment priming has been investigated mainly as an intervention for behavioral outcomes such as increased positive affect and decreased negative affect, lowered stigmatization of outgroup members, increased empathy, and improved emotional regulatory functioning (see Rowe, Gold, \& Carnelley, 2020, for a review). It has also been used to guide understanding of the internal working model that attachment systems employ (Gillath, Karantzas, \& Fraley, 2016). Theoretically, it is understood that when activating the secure attachment schema under threat, the regulation of the threat response frees up cognitive resources for normal functioning and exploratory behavior (Gillath et al., 2016, Mikulincer \& Sheffi, 2000). Historically, the methods of security priming have involved the implicit presentation of attachment-related images or words, an explicit task involving guided imagery or reading scenarios, or a combination of both implicit and explicit tasks in a repeatedpriming paradigm (Rowe et al., 2020).

Secure attachment priming works by overriding the dispositional attachment system and therefore is an ideal intervention for those with an insecure dispositional attachment style, as these populations may not have ideal regulatory mechanisms otherwise. It is also a useful intervention for more securely attached 
individuals in scenarios of non-attachment related threat, as it can facilitate the threat regulation process in situations where it is not usually activated. The mechanism by which secure attachment priming facilitates improved outcomes has been through decreased threat appraisal (Mikulincer \& Shaver, 2001), accomplished for insecurely attached populations through attention redirection, heightened social emotional processing, more positive evaluation of stimuli, and a general more positive emotional state (Bai et al., 2019; Canterberry \& Gillath, 2013; Tang et al., 2017). Secure attachment priming in one-shot priming procedures has also been used to enhance performance on cognitive tasks like creative problem solving (Mikulincer, Shaver, \& Rom, 2011) and working memory tasks, though dispositional attachment style and stimulus type (e.g. attachment-related or non-related pictures) interacted with the efficacy of the prime in these experiments (Bai et al., 2019). Studies which used a repeated-priming paradigm, whether using congruent (e.g. implicit-implicit) or mixed (e.g. implicit-explicit) priming designs, found a stronger and longer-lasting effect of the security prime, as well as a more consistent effect without the moderation by dispositional attachment styles (Rowe, Gold, \& Carnelley, 2020). Therefore, it is more beneficial to use a repeated-priming paradigm whenever possible to ensure the efficacy of the prime.

It has been well-established that security priming can reduce negative evaluations of outgroup members as well as reduce both the imagined and enacted harm towards an outgroup member (Mikulincer \& Shaver, 2001; Saleem et al., 2015). Security priming, however, has not been investigated in relation to early perceptual influences impacting those attitudinal and behavioral outcomes. It is theorized that security priming improves perceptions of outgroup members due to its effect of enhancing exploratory functioning (e.g. Mikulincer \& Shaver, 2001), but a proper mechanism has not been identified. From neural-imaging research we know that security priming can improve the emotional processing of positive and negative face stimuli and mitigate negative affect when perceiving those stimuli (Tang et al., 2017). This soothing effect can then help improve performance on tasks which require more cognitive resources (working memory performance; Bai et al., 2019). But how does security priming influence the perception of faces, and particularly the perception of race in faces? Two main questions drive the connection between security priming's effects on perception and its effects on ingroup/outgroup evaluation: Is it 
possible for a security prime to influence the perceptual processes that lead to phenomena such as the cross-race effect, where outgroup faces receive diminished processing? And could a security prime mitigate the tendency for hypodescent, where we categorize a racially ambiguous face as its minority/outgroup race? The answers to these questions could determine whether security priming can be used as an effective intervention to reduce the likelihood of an eyewitness misidentifying a suspect of another race in a lineup (Dodson \& Dobolyi, 2016). This potential intervention could even promote more fluid conceptions of race, an important motivator for increasing emotional engagement in issues of racial inequity (Williams \& Eberhardt, 2008). To understand the plausibility of a security prime impacting the cross-race effect and race categorization, we first need to understand the process of face perception and the social biases present in that processing.

\section{Face Perception Processing Biases}

At the very first stages of processing when we see a face, the way we process that face is already prone to social biases like ingroup-outgroup identification (e.g. Cassidy et al., 2014). The cross-race effect is one phenomenon that demonstrates the effects of early ingroup-outgroup identification. The cross-race effect occurs in memory tasks where participants have more difficulty accurately recognizing the faces belonging to an outgroup race compared to faces of their congruent race. This leads to trouble distinguishing the faces of other races and has ultimately been connected to the rampant misidentification of disproportionately Black suspects during eyewitness trials (Reinitz, 2018).

The cross-race effect has been supported robustly in populations which have lived in racially homogenous environments, though the limited research in multiracial and multicultural populations (SE Asian Archipelago nations) has found that in minority race groups, faces of the predominant racial group are not subject to the cross-race effect (Estudillo et al., 2020; Tham, Bremner, \& Hay, 2017). For the main body of research with white and East Asian participants, some of the factors which moderate the crossrace effect are when faces are presented in a way that allows participants to see multiple angles, and the cross-race effect is mitigated (Zhao et al., 2014); prior expertise or training in individuating other-race faces also mitigates the cross-race effect (Stahl et al., 2008); and motivation to individuate faces by being 
informed of the cross-race effect mitigates the cross-race effect (Hugenberg et al., 2007). But the most effective moderator of the cross-race effect is social context, and especially the tendency to categorize people into ingroups and outgroups (e.g. Hehman, Mania, \& Gaertner, 2010). Relevant social context, such as priming one cultural identity over another, has consistently moderated the cross-race effect in mono- and multi-cultural populations where they process faces of the ingroup better despite the race of that face (e.g. Marsh, Pezdek, \& Ozery, 2016).

The impact of ingroup/outgroup identification on the cross-race effect can be explained through processing biases that occur due to this categorization. In face/object recognition research, holistic processing refers to the processing of the relations between features rather than of the features themselves and is the fastest and most efficient form of processing that is usually reserved for faces or other things we have expertise in distinguishing. However, experimentally- and naturally-induced categorizations of ingroup and outgroup faces seem to cause differential processing of faces, where outgroup faces have reduced holistic processing and therefore reduced quality of information extraction (Cassidy et al., 2014; Hehman, Mania, \& Gaertner, 2009). It is most commonly theorized that a lack of face expertise is the cause for this deficiency in processing of outgroup faces, where increased exposure to and consequently the need to often individuate outgroup faces mitigates the cross-race effect in experimental contexts (Gaither et al., 2012; Stahl et al., 2008). Reduced holistic processing is indicative of lower quality information extraction, which could provide an explanation for the cross-race effect and the resulting lower recognition for outgroup faces. However, it is also possible that this deficiency in holistic processing, but not other behavioral outcomes of own-race bias like sensitivity to featural differences, is unique to white populations: Comparisons of Chinese and white populations from China and USA, respectively, revealed reduced holistic processing for other-race faces only in the white population. Both populations, however, evidenced own-race bias in a variety of facial individuation tasks (Mondloch et al., 2010).

It seems that reduced holistic processing is not the sole contributor to the cross-race effect, and other motivational or processing factors may be contributing to the effect as well. While not studying 
holistic/configural processing directly, Dickter \& Bartholow (2007) found, through event-related potential (ERP) in a sample of both Black and white participants, that an ingroup-race face received enhanced processing overall, while an outgroup-race face elicited neural responses indicative of surprise or potential threat. Attempting to draw attention away from race, gender was made salient as the ingroup, but the ingroup-outgroup effect for gender was not seen. Rather, the brain still responded to the racerelevant dimension instead of the gender dimension. In other words, making gender salient instead of race did not override the initial perception of racial ingroup membership and its neural correlates (Dickter \& Bartholow, 2007). The implications of these finding are that perceived racial outgroup membership likely dominates the early social categorization processes. Dickter and Bartholow's findings also clarified that it was the categorization of ingroup faces, not of outgroup faces, that led to processing advantages for ingroup faces: reaction times for participants completing a flanker task were significantly impacted by their neural response to ingroup faces but not by their neural responses to outgroup faces. Thus, it is likely the recognition of ingroup targets that encourage enhanced (and, perhaps, holistic) processing, rather than having dulled processing when viewing outgroup targets. These findings are in line with the hypothesis that the cross-race effect occurs due to perceptual expertise in the processing of same-race faces. And though Dicker and Bartholow found that making gender salient did not impact processing in terms of gender ingroups, this finding does not rule out the possibility that social contexts outside of race can impact the cross-race effect.

Investigating further the relation between the cross-race effect and social context, in studies with social manipulations of ingroups (e.g. university affiliation), faces were processed more holistically despite their racial categorization if they were reinforced as an ingroup member, and conversely outgroup faces were processed less holistically even if they were of a congruent race (Hugenberg \& Cornielle, 2009). Additionally, reinforcing university affiliation as an ingroup led to race having no significant effect on recall (Hehman, Mania, \& Gaertner, 2010). However, a separate study found that for all white participants, ingroup identities such as economic status and university affiliation impacted recall only for white outgroup faces, not for ingroup or outgroup Black faces (Shriver, Young, \& Hugenberg, 2007). The 
difference in these findings is likely due to the methods involved: Hugenberg and Cornielle (2009) presented eight faces separated either by university affiliation or by race to make the ingroup cue salient; whereas Shriver, Young, and Hugenberg presented the faces singly in a standard old or new face recognition task. Therefore, it appears that explicit task goals involving salient ingroup cues can impact facial recall to mitigate the cross-race effect, but without these non-racial ingroup cues the processing and recognition of outgroup race faces remains prone to the cross-race effect (in white populations). From the early processing advantages of ingroup race found in Dickter and Bartholow (2007) to the reemergence of the cross-race effect when no social ingroup cue is made salient (Shriver, Young, \& Hugenberg, 2007), perception of race seems to be a default attentional bias for face perception and processing in these experimental scenarios, revealing a potentially manipulable dimension that security priming, a technique which redistributes attentional resources, may be able to influence.

Closely linked to the cross-race effect, the second main phenomenon stemming from early ingroup/outgroup processing bias is racial categorization, specifically for racially ambiguous faces. As established earlier, the categorization of race occurs during early attentional processes with little effect by explicit task goals (Dickter \& Bartholow, 2007). For white, Westernized populations, if they strongly identify with their ingroup then they tend to categorize racially ambiguous faces as an outgroup race more often than an ingroup one (Castano et al., 2002; Knowles \& Peng, 2005). And this is also highly impacted by social motivation, where social exclusion can motivate the categorization of racially ambiguous faces to be Black for both white and Black participants (Gaither et al., 2016). This finding that both Black and white participants rated the ambiguous face as being black was from a study with an American university population and can potentially be explained by the construct of racial essentialism, as Gaither et al. suggest in their discussion.

\section{Racial Essentialism as a Moderator}

Racial essentialism represents the belief that race is biologically based and unchanging, and it is usually associated with more rigid conceptions of race (Williams \& Eberhardt, 2008). For an American population, this thinking may have originated from the "One-Drop Rule," a law upheld in the Jim Crow 
era that a drop of African American blood made an individual Black. This belief is still present today and may be what is influencing the categorization of racially ambiguous faces as Black (Gaither et al., 2016). In studies with white, Western populations, bias to categorize ambiguous race faces as an outgroup race emerge most when identity is threatened, if one has more negative attitudes towards outgroup members, and if one holds stronger beliefs in racial essentialism (Gaither et al., 2014; Ho, Roberts, \& Gelman, 2015; Chao, Hong, \& Chiu, 2013). Racial essentialism predicted hypodescent of an ambiguously multiracial individual when attitudes towards Black people were more negative among white participants (Ho, Roberts, \& Gelman, 2015). Even in a population of white children, racial essentialism was associated with higher recognition of white faces, lower recognition of Black faces, and the lowest recognition of racially ambiguous faces (Gaither et al., 2014). Racial essentialism may even emerge as an interacting factor with the cross-race effect and facial processing, though there is evidence for its role in both reducing and improving ambiguous face processing, perhaps dependent on the race of the participant. Biracial (Asian/white) participants who held lower scores in racial essentialism performed much better on a facial memory task in all conditions (ingroup, outgroup, and ambiguous) compared to monoracial participants (Pauker \& Ambady, 2009). In a different study, monoracial participants with higher scores of racial essentialism were more sensitive to race-specific face features and were more accurate in discerning between face stimuli which differed subtly in those features (Chao, Hong, \& Chiu, 2013). These mixed findings present an opportunity to further clarify how racial essentialism may play into face processing that contributes to the cross-race effect, and additionally how this interaction may be impacted by an activation of the secure attachment schema through priming.

Fluid social cognition, in direct opposition to racial essentialism, led participants to have less essentialism and rate racially ambiguous faces as multiracial rather than monoracial more often when given the choice between the two (Slepian et al., 2014). In this scenario, fluid social cognition was primed through fluidity in movements (tracing, or watching someone trace, a rounded flowing pattern as opposed to a rigid, angular pattern). Thus, primes mitigating the effects of racial essentialism on categorization exist, suggesting that racial essentialism is mutable and could potentially be impacted by a security prime. 
Mikulincer and Arad (1999) found a related effect of security priming, where it increased participants' openness to belief-discrepant information. Therefore, it is plausible that security priming can influence racial essentialism by increasing fluid social cognition as well.

\section{Security Priming's Impact on Processing Biases}

It is, however, unknown whether and how security priming impacts racial essentialism, as well as how security priming may impact the early processing biases caused by ingroup-outgroup perception based on race. Findings from Boccato et al.'s (2015) study on attachment security and intergroup contact suggest that those with attachment security, in general, have higher quantity and quality of contact with racial outgroup members, factors which are negatively correlated to feelings of racial essentialism and outgroup derogation. This study will be the first to test for security priming's direct influence on racial essentialism and the early processing biases occurring due to racial ingroup-outgroup categorizations. Parallel research in priming has found an impact of the prime on the processing cues of faces: there is evidence that mortality salience priming, a security-related prime with the opposite effect of security priming, enhanced the processing of ingroup cues in angry ingroup and outgroup faces, all of which occurred at the early stages of processing ( 200-300ms after presentation; Henry, Bartholow, \& Arndt, 2010). Where mortality salience priming heightens the threat cues of stimuli, security priming regulates the threat appraisal (Bai et al., 2019; Canterberry \& Gillath, 2013; Mikulincer \& Shaver, 2001). This points to the plausibility of a security prime impacting processing and categorization cues when presented with faces, as attachment research has also determined that security priming operates at around 200 to 300 milliseconds after presentation (e.g. Bai et al., 2019), the same early stages of processing that ingroupoutgroup and racial categorization have been found to occur (Dickter \& Bartholow, 2007). Using fMRI, Tang and colleagues (2017) found that in comparison to anxiously attached participants, securely attached participants had increased processing of faces in the visual association area V2, commonly associated with feature extraction. This finding suggests the potential for a security prime to facilitate feature extraction in faces which may mitigate the cross-race effect. 
This study utilized a multidimensional scaling (MDS) framework to examine the categorization biases that are caused by early racial outgroup processing. This MDS framework is frequently employed in cross-race effect research as it is able to represent the latent dimensions used to categorize and recognize the infinite variations in faces.

\section{Using MDS for an Individual Differences Model}

MDS is an exploratory procedure that provides a multidimensional representation of the structure with which a person perceives a stimulus. The present study gauged participants' implicit perceptual structures by having them make similarity judgements between all pairs in a set of stimuli. Following an MDS framework in cross-race effect research usually implies the acceptance of the multidimensional face space hypothesis, which postulates that the way in which faces are perceived and sorted in our memory is congruent to the Euclidean space used in MDS, where faces with high perceived similarity cluster closer together. Arguments of this framework directly reflecting the architecture of the mind aside, it has continuously provided a robust model of the cross-race effect, represented in this framework as other-race faces clustering more densely together than the same-race faces, despite the objective similarities between the respective group members being equal. It is important to note that research into the cross-race effect, especially research which used an MDS framework, has been investigated primarily white populations. Papesh and Goldinger (2010) found support for the multidimensional face-space hypothesis with Black and White face stimuli, where in white participants the distances between Black faces were significantly smaller than between White faces. This study included only $3 \%$ Black participants, so there were no separate analyses of the cross-race effect by race. The faces used in Papesh and Goldinger were generated from a prototype White face with a "genetic randomness" tool to change facial features, and skin tone was assigned to the faces based on a counterbalance across participants. This approach allowed for the comparison of objective similarity of face features with perceived similarity, revealing that skin tone was the main factor influencing the cross-race effect rather than facial features alone (Papesh \& Goldinger, 2010). 
The present study used MDS to examine the individual differences model across participants. The individual differences model allows for the identification of superordinate perceptual dimensions that are shared across groups of individuals, so we are able to compare group (e.g. secure prime vs neutral prime) differences in perceptual dimensions, individual differences, and superordinate (latent) group differences that may have otherwise been obscured by averaging across experimental groups. The distances between each stimulus can then be visualized on a plane (Euclidean space) where the stimuli rated as most similar are clustered together. These clusters are then analyzed for latent factors that influenced how the participants determined the categorizations. An example factor could be skin tone (Papesh \& Goldinger, 2010). Using MDS in the present study was attractive because of its implicit nature, where participants could make categorization judgements without being explicitly instructed to focus on race or any other dimension unequally. For example, we can study how changes in attachment security potentially influence people's categorizations of faces. A difference across experimental groups would point to a mechanism in security priming that is influencing perceptions and/or categorizations of individual faces that could later be influencing their attitudes towards outgroups.

\section{Purpose of the Present Study}

The perceptual-processing impacts of security priming have been established for general attachment-threat scenarios, where the security prime redistributes attention for lowered threat appraisal and engenders more positive appraisal of stimuli (Bai et al., 2019; Canterberry \& Gillath, 2013). For ingroup/outgroup research, however, only the overt attitudinal and behavioral impacts of the security prime, reducing outgroup derogation and harm (e.g. Saleem et al., 2015), have been established. Intergroup research with security priming has not connected the attitudinal and behavioral outcomes to the perceptual mechanism in the security prime. To get at this connection, this study identified ingroup category formation and racial categorization as two processes that occur early on in processing that could have a bearing on future outgroup interactions. Racial essentialism was also measured because of its effect on the categorization of racially ambiguous individuals, as well as the potential for a security prime to impact it. The present study identified whether and how repeated security priming influenced the 
perception of same and other race faces in a multidimensional scaling (MDS) paradigm, which measures the categorization structure for faces ranging in their racial ambiguity. It was predicted that one of the structural biases detected from the MDS analysis would be the cross-race effect, especially because presentation of the static, single-perspective faces and the similarity rating task are the factors that bring about the cross-race effect most strongly (Zhao et al., 2014). Also, considering the early processing bias of race (e.g. Dickter \& Bartholow, 2007), without an overt task goal it is likely that race would be the main factor participants use to categorize faces. Additionally, by examining racial essentialism and taking direct measurements of perceived race, it was also possible to determine if racial essentialism served as a mediator or a moderator of security priming's impact on racial outgroup perception.

\section{Hypotheses}

Due to the exploratory nature of MDS and the resultant dimensions, I first aimed to confirm the presence of a race dimension to conduct further analyses regarding that race dimension. There is evidence to support that one of the main dimensions to manifest from this MDS framework would be a race dimension; for instance, the findings that without an overt task goal, race was processed early on and used in categorization (e.g. Dickter \& Barthalow, 2007) and that skin tone was a main dimension of categorization when other facial characteristics were controlled across skin tones (Papesh \& Goldinger, 2010).

Given the presence of a race dimension, the impact of security priming on the early processing of visual stimuli, such as lowered threat appraisal and heightened social emotional processing (Bai et al., 2019; Canterberry \& Gillath, 2013), was predicted to influence the participants' categorization structures in two ways: (a) the weight of the perceived race dimension would be lower for the security prime group than the neutral prime group as pre-priming racial essentialism was lower, and (b) the distribution of dimension weights would be different for the security prime group compared to the neutral prime group as pre-priming racial essentialism was lower. I predicted that security priming would mitigate the degree to which the cross-race effect was observed when racial essentialism was lower, where the cross-race effect was captured in three different ways: First, it was compared on the group-level by comparing the 
average Euclidean distances between same-race faces for the secure participants' general face-space and the neutral participants' general face-space; Second, it was approximated by a difference in dimension weights, where lower reliance on dimensions that individuate facial characteristics would indicate higher cross-race effect; And lastly, it was compared at the individual level by computing the variation in similarity ratings between same-race faces for all participants. Additionally, I hypothesized that the security prime group would have lower scores of racial essentialism post-prime because of security priming's ability to increase resources for cognitive flexibility (e.g. Mikulincer \& Arad, 1999). Lowering racial essentialism in this way would also lead to average ethnicity ratings of all faces to be closer to White than Black in the security prime group as participants acknowledge multiracial qualities (e.g. Slepian et al., 2014).

Lastly, I hypothesized that racial essentialism would predict the perceived Blackness of the target stimuli, where, in all participants, higher essentialism would result in higher ratings of Blackness on the slider task for ambiguous targets. I investigated this effect directly to confirm that the construct of racial essentialism does correlate to the construct of perceived race for external validity. Investigation of these hypotheses clarified the unknown connections between security priming and essentialist thought, as well as the impact of security priming and essentialist thought on the implicit categorization of Black and White face stimuli. 


\section{CHAPTER III: METHODOLOGY AND RESULTS}

The experiment was administered in an entirely online format due to the circumstances surrounding COVID-19 and to ensure the safety of both participants and experimenters. As such, this study also served as validation for the online administration of Mikulincer, Shaver, and Rom's (2011) guided imagery task which explicitly primes secure attachment, Cassidy et al.'s (2009) implicit security priming paired-comparison task, and a test of the feasibility for the online administration of pairedcomparison tasks used in MDS analysis, with overall positive results.

\section{Participants and Recruitment}

Students from Illinois State University were recruited via mass email to the student body or through the SONA system, the university's research participation management system, resulting in over 400 responses to the first part of the study. The target response rate was 107 participants, and data collection was halted after collecting 150 complete responses. Of the 446 initial respondents, 323 (72\%) completed Session one and 150 (34\%) finished both parts one and two. Only the participants who completed both parts were considered in the analyses, yielding 108 (69.7\%) white, 11 (7.1\%) Black, 8 (5.2\%) Asian/Pacific Islander, and 20 (12.9\%) Hispanic/LatinX participants. Other demographic attributes such as gender were not collected.

Random assignment to secure or neutral priming groups occurred through Qualtrics. Eligible participants were anyone 18 years of age or older who had consistent access to a computer and keyboard, internet, and who were not visually impaired. Ineligibility arose if the participant could only use a phone or tablet, at which point the site hosting the experiment (Psytoolkit) redirected them until they switched to a computer. 


\section{Measures}

\section{Attachment}

The Relationship Structures (ECR-RS; Fraley et al., 2011) questionnaire is a measure of attachment for a variety of relationship domains. It uses the same 9 items to assess attachment in romantic, parental, and close other domains, typically assessed with the targets of mother, father, romantic partner, and best friend. However, the scale was designed to be adaptable to a large variety of targets, like God or a pet, and can also allow for participants to decide their own relational targets. Example items include "I find it easy to depend on this person," and "I'm afraid this person may abandon me." Items are scored on a Likert scale from 1 (strongly disagree) to 7 (strongly agree).

Though not as reliable as the original ECR-R scale it was developed from, the ECR-RS has shown a test-retest reliability over the span of 30 days for romantic relationships of .65 and a test-retest reliability in the parental domain of .80 . The internal consistency is excellent for measuring global attachment ( $\alpha=.81$ to .85 ; Fraley et al., 2015) and for measuring domain specific attachment ( $\alpha=.87$ to $\alpha=.92$; Fraley et al., 2011). It has also shown construct validity with the ECR-R in measuring attachment structures (Fraley et al., 2011), criterion-related validity with relational outcomes (Fraley et al., 2011), and has been validated in populations of university students and adolescents aged 15 to 18 (Fraley et al., 2011; Donbaek \& Elklit 2014). Due to its measurement of multiple attachment structures, the ECR-RS can potentially have less internal reliability if used as a measure of global attachment and lower test-retest reliability than other common measures of attachment. However, it does not require the exclusion of participants who are without past romantic relationship experience, an element that has been chronically biasing samples in adult attachment research using the ECR or ECR-R which ask questions directly referring to a past romantic relationship partner. In the interest of the present study, which included those in a university population who may not have had romantic relationships, this measure served as an inclusive, reliable, and succinct measure of general attachment.

The ECR-RS was used in this study to measure general global attachment, with the instructions to participants being "Please read each of the following statements and rate the extent to which you believe 
each statement best describes your feelings about close relationships in general.” Internal consistency was $\alpha=.76$.

\section{Racial Essentialism}

The Race Conceptions Scale (RCS, Williams \& Eberhardt, 2008) is a 22-item measure of racial essentialism, addressing examinee attitudes towards race as being biological, natural, stable over time within societies, contexts, and individuals, and discernable by observation. The RCS captures a related but separate construct from prejudice and racism. It has shown high internal reliability in US populations and in a variety of study designs ( $\alpha=.73$ to $\alpha=.93$, e.g., Donovan, 2014, Pauker et al., 2018), and is robust to social desirability, partly due to the scale's focus on neutral beliefs about race (Williams \& Eberhardt, 2008). Scores can range from 22 to 154; high scores on this measure indicate higher agreement with statements like "Race is biologically determined" and "A person's race is fixed at birth." For the current study, internal consistency of the RCS for Session 1 was $\alpha=.82(N=148, M=90.00, S D=15.81)$, and for Session 2 was $\alpha=.85(N=143, M=89.3, S D=17.46)$. The scale also showed high test-retest reliability despite the prediction that the scores would change due to secure priming, $r=.88$.

\section{Felt Security}

The Felt Security Scale (Luke et al., 2012) is composed of 16 items that assess to what extent participants feel comforted, supported, looked after, cared for, secure, safe, protected, unthreatened, better about themselves, valued, more positive about themselves, loved, cherished, treasured, adored, and the extent to which they really like themselves. Multiple studies have corroborated the FSS's ability to capture a difference in felt security between secure attachment and neutral prime conditions, with the security prime accounting for $15.5 \%$ (Carnelley et al., 2018) to $40 \%$ of the variability in felt security (Carnelley et al., 2015; Otway et al., 2014). The scale was created for a study (Barts \& Lydon, 2004) in which felt security was measured shortly after a priming procedure, and has continued to be used in the same context of primarily white, majority female British populations taking the FSS immediately after a Bartz and Lydon explicit prime. Those studies which assessed felt security after a Bartz \& Lydon prime have had excellent internal reliability ( $\alpha=.96$ to .99; Carnelley et al., 2015; Carnelley et al., 2018; Luke 
et al., 2012; Otway et al., 2014). One study which found lower reliability ( $\alpha=.84$ to .88) administered a shorter 9-item version of the FSS after a different experimental manipulation and in a population more diverse in age, ethnicity (Jakubiak \& Feeney, 2016). The current study FSS had an internal reliability of $\alpha$ $=.97$.

\section{Perceived Race}

The perceived race scale was developed for this study to capture how the participant perceived the race of each Black, White, or Ambiguous face in a continuum from 0 (Black) to 100 (White). Its continuous nature was assessed by graphing the frequency distribution of the average face ratings collected from all participants, revealing the expected tri-modal distribution with adequate variation around each category point (Black, Multiracial, and White) to constitute a continuous, rather than categorical, measure (Figure A1).

\section{Ingroup Identification}

The Inclusion of Ingroup in the Self (IIS; Tropp \& Wright, 2001) measure was used to capture the participants' identification with white and Black identities. IIS is a one-item measure where participants choose which out of seven Venn diagram pairs best represents their identification with a group. One circle represents the self, the other represents the group. Each of the seven pairs is increasingly intertwined (Figure A2). The IIS has shown a test-retest reliability of .76 along with concurrent and discriminant validity in racially diverse samples (Tropp \& Wright, 2001).

\section{Demographic Questionnaire}

Due to the nature of the study being centered on racial perceptions, it was crucial to measure participants' ethnicity and their estimated contact with white and Black individuals as these experiences may have interacted with their perceptions of outgroups. Participants could type up to four ethnicities which they identify as and were subsequently asked to complete the IIS for Black and white identities. Afterwards, participants answered four questions pertaining to contact with Black and white individuals: how often they interacted with that ethnicity in high school and in college, rated on a three-point scale 
from Rarely to Frequently; and whether (no/yes) they have had a teacher, relative or friend with that ethnicity.

\section{Materials}

\section{Explicit Priming}

Rowe et al. (2020) found in their meta-analysis that priming tasks which require participants to process information about their own attachment experiences are most effective, thus, this study used a guided imagery task which facilitates that form of attachment processing. Adapting the procedure from Mikulincer, Shaver and Rom (2011) to an online format, participants in the security prime condition were asked to think of a person to whom they turn when feeling distressed or worried. They listed six central qualities of that person, visualized a specific time when that person provided comfort in a time of need for at least one minute, then typed a description of the imagined scenario and the feelings felt during it.

In the neutral priming condition, participants thought of someone they know but are not close with, listed 6 physical traits of that person, visualized a specific activity (e.g. lecture) they attended with that person, then typed a description of that scenario and the feelings felt during it. All participants were ensured that their responses would remain completely anonymous and they were to describe in only as much detail as was comfortable.

\section{Implicit Priming Training Task}

Participants were implicitly primed during the 20-trial training paired-comparison task through words shown between each trial of the task, programmed and presented with PsyToolkit 3.1.1 (Stoet, 2010, 2017). The initial landing page of the experiment informed the participants of the estimated length of the similarity rating task and instructed them to find a quiet, distraction-free area before electing to start the experiment. After briefing participants on the format of the similarity rating task, their browser was made full-screen and a picture was displayed to guide the participants in how they were to place their hands on the keyboard so that they had fingers on each number from one to eight. Before the task began, participants were shown instructions about making ratings from 1 to 8 , then instructions to endeavor to make their ratings in under 5 seconds. All instructions were self-paced. 
Once the task began, participants saw a white background with the forward mask "XXXXXXXX" in the center for 700 milliseconds. A prime word was displayed for 20 milliseconds before switching to the backward mask of "XXXXXXXX" for 400 milliseconds. Participants were subliminally exposed to prime words from either the secure or neutral condition depending on their random assignment. The secure attachment group was administered the words "SAFE," "NURTURE," “WARMTH," and "COMFORT” from Cassidy et al.'s (2009) implicit security priming study. The neutral words "BATH," "BLENDER," "NUMBER" and "VEHICLE" were chosen to match the length of the words shown to the secure group. Participants were then shown two female ambiguous-race faces on opposite ends of the screen with the scale of 1 (Least similar) to 8 (Most similar). After five seconds, the message "Please make your selection as quickly as possible" displayed at the bottom of the screen. Participants who failed to make any rating after 10 minutes would be keyed as 0 , but no participants encountered this scenario.

\section{Face Stimuli}

The face stimuli used were a 20-item subset of Black, White, and ambiguous Male faces with neutral expressions from Pauker et al.'s (2009) set of 40 computer generated faces. The faces were cropped just below the hairline and presented at $291 \times 366$ pixels. The original 40 male and female faces were rated reliably by 17 white and 9 Black individuals for their prototypicality in terms of race $(\alpha=.92)$, attractiveness $(\alpha=.94)$, and distinctiveness ( $\alpha=.94$; Pauker et al., 2009). As expected, the ambiguous faces were rated as less prototypical than the Black and White faces, and all three groups of faces were rated equivalently in attractiveness and distinctiveness (Pauker et al., 2009). In addition, the ambiguous faces were equally likely to be categorized as Black as they were White (Pauker et al., 2009). Unfortunately, the original data from these analyses were not available for reanalysis using the 20 faces chosen for the current study. 


\section{Figure 1}

Example Face Stimuli
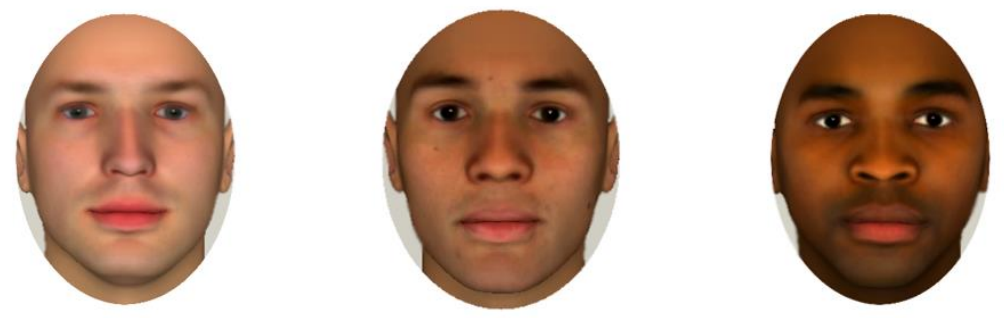

Note. White, Ambiguous, and Black male face stimuli. Generated by Pauker et al. (2009) using FaceGen

Modeler 3.1.

\section{Procedure}

Participants were able to begin Session 1 by either following a link from the mass email or by following the link in the SONA system, where they were then redirected to Qualtrics. The online form in Qualtrics prompted participants before and after the consent form to find a private space where they would be able to work without distraction for up to 15 minutes. An initial screening question ensured that they had access to a computer with a keyboard. After providing consent, confirming they had access to the necessary supplies, and confirming that they were working without other distractions, participants completed in a randomized order measures of attachment (ECR-RS) and racial essentialism (RCS). Once completed, the form prompted them to follow the onscreen instructions for a guided visual imagery task. During this visual imagery task, participants were asked to either visualize a person who helped them in times of need (the secure prime group) or an acquaintance (the neutral prime group). This part of the task lasted about one to five minutes. Afterwards, participants were prompted to type a summary of the person whom they visualized, including the feelings they had during the task. Participants then completed the Felt Security Scale, marking the end of Session 1. Most participants took less than 10 minutes to complete Session 1. 
Session 2 took place two to six days following the first session. Participants were sent an email 24 hours after they entered the first session that either contained a link to the second session, or a reminder to $\log$ into SONA to complete the second session. Participants were shown a disclaimer that the next task could take from 30 minutes to an hour, and that they could only proceed if they were using a computer or laptop with a keyboard. Upon agreeing to the terms, following the link in the Qualtrics survey opened a new window to the experiment programmed and hosted in PsyToolkit.org (Stoet, 2017). For all the training, practice, and experiment trials, faces were presented on the screen in pairs, each face about $4 \mathrm{~cm}$ apart (500 pixels from center to center). Participants first completed the training task which functioned as the implicit prime. As a check to ensure the primes were subliminal, for the last 5 trials of the training task participants were told that words were going to be presented between trials and that they were report the word they saw, if they saw anything (Bargh \& Chartrand, 2000). After the training task, participants completed a 20-trial practice task, where they made comparisons of ambiguous, White, and Black male faces not used in the experimental task but sourced from the same subset of faces from Pauker et al. (2009). Between each trial there was the same fixation point "XXXXXXXX" from the training trials, but participants were not primed with any words. This clearing of the screen with a fixation point was necessary between trials to prevent the face distortion effect occurring from the fast presentation of faces aligned at eye-level (see Tangen, Murphy, \& Thompson, 2011).

For the experimental task, participants compared every combination of face pairs in 190 trials with 10 repeated trials at the end for an internal consistency check, totaling to 200 trials. There were five blocks with 40 trials each. Between each block, participants were shown a break screen that allowed them to press space when they were ready to continue with the next block. Break times were recorded. Each participant rated the faces in the same order, which was optimized for paired comparison with an even number of stimuli (Wells, 1991). The entire experiment, from implicit prime to finishing the 200 ratings, took participants 20 minutes on average to complete. There was a small set of participants who completed the task over the span of 10 hours. 
After completing the paired comparison task for all face pairs, participants were redirected to Qualtrics where they then began a perceived race slider task. Each of the 20 faces were presented with a continuous slider to indicate how Black or White a face appeared with the prompt "Please use the slider below to indicate the race of this person." "Black" and "White" were on opposite ends of the slider. "Multiracial" appeared in the center of the slider. Once completing the slider task, participants completed the RCS again, then answered the demographic questionnaire and the ISS for Black and white ethnicities. Finally, participants reached the debriefing page which provided a viewable attachment to explain the implicit priming nature of the study and inform participants of the option to opt out while still receiving credit/compensation. Many participants completed this portion of the experiment a day or more after completing the similarity ratings or did not complete this portion of the experiment at all.

\section{Hypotheses}

The hypotheses for this study were contingent upon a perceived race dimension being observed from the MDS solution and were the following:

1. Racial essentialism scores would predict the perceived Blackness of the target stimuli, where higher RCS scores would be associated with higher scores of Blackness for racially ambiguous targets. This hypothesis was to confirm that the construct of racial essentialism would correlate to the construct of perceived race for external validity.

2. One of the main dimensions participants use to categorize the faces would be a perceived race dimension.

3a. The security priming group would demonstrate lower level of the cross-race effect, indicated by 1) larger average distances between Black face pairs compared to the neutral prime group $(3 a .1), 2)$ by an interaction between face race and prime condition where the secure prime group would exhibit less reliance on a race dimension and more reliance on a dimension using facial features compared to the neutral prime group for Black faces, indicated by dimension weights (3a.2), and finally 3 ) by lower variation in similarity ratings between Black face pairs (3a.3). 
3b. Racial essentialism would moderate the effects of the security prime on dimension weights, where participants with higher racial essentialism would show higher reliance on a race dimension despite the priming condition, mitigating the impact of the security prime.

4. The security prime group would have larger decreases in racial essentialism post-prime.

5a. Average ethnicity ratings of ambiguous faces on the perceived race slider task would be closer to 50 or higher (on a scale of $0=$ Black to $100=$ White) for the security prime group, indicating less hypodescent, coupled with greater variations in ratings of Black faces.

5b. Average racial essentialism scores would moderate the effect of security priming on the scores for the perceived race slider task for Black, White, and Ambiguous faces. Higher racial essentialism scores would predict more monoracial ratings (sliders closer to extreme 0 or 100 ends) for all three face types.

If a race dimension was not observed in the MDS solution, the main hypothesis was that the security priming group would have significantly different distributions of dimension weights compared to the neutral priming group, indicating that security priming was impacting the way participants were making category judgements for White, Black, and Ambiguous faces. 


\section{CHAPTER IV: RESULTS}

\section{Data Treatment}

Missing items for the ECR (attachment), FSS (felt security), and RCS (racial essentialism) were treated with multiple imputation to generate possible values for missing values with the expectationmaximization (EM) method for participants who skipped some items; missing values were not replaced for participants who skipped the entire measure $(n=7)$. Three missing values were replaced across the RCS at time 1, 6 values were replaced across the RCS at time 2, and 4 values were replaced across the ECR. The EM iterative method computes expected values based on a distribution made from existing values, replaces those values, and recomputes expected values until the changes between iterations become negligible. EM imputation is far superior to the mean-substitution and listwise deletion methods when these data are to be used in regression analysis, as mean-substitution will ignore a participant's pattern of scores and listwise deletion greatly reduces sample size (Musil et al., 2002). EM imputation is only appropriate if the missing values are not due to an underlying pattern that may bias the data. Little's MCAR (missing cases at random) test was not significant for the RCS at time $1\left(\chi^{2}[42, \mathrm{~N}=150]=52.56\right.$, $p=.13)$, time $2\left(\chi^{2}[104, \mathrm{~N}=148-150]=100.18, p=.59\right)$, or for the $\operatorname{ECR}\left(\chi^{2}[24, \mathrm{~N}=148-150)=33.64, p\right.$ $=.09$ ), indicating that the values were missing at random and EM imputation was an appropriate procedure to handle the missing cases.

\section{Preliminary Analyses and Hypotheses Testing}

This study examined whether attachment security priming decreased the use of race to make category judgements in the perception of faces (Hypothesis 2), decreased racial essentialism and the tendency for hypodescent of ambiguous-race faces (Hypotheses 4-5a), and decreased the cross-race effect (Hypothesis 3a). The study also examined whether racial essentialism interacted with security priming to affect the use of race in category judgements and the cross-race effect (Hypotheses 1, 3b and 4b). The cross-race effect was operationalized in three ways: (a) the average distances between Black faces being smaller compared to White or Ambiguous faces, compared at the group level; (b) a difference in dimension weights with high reliance on a race dimension alongside low reliance on dimensions 
individuating facial characteristics, compared at the individual level; and (c) lower variation in similarity ratings between same-race faces accompanied by high average similarity ratings, compared at the individual level (Hypothesis 3a). It was necessary to define operational definitions at the group and individual level because of the nature of the MDS output; the standard metric for the cross-race effect, Euclidean distances between same-race faces, could only be generated as an average across participants and therefore could not be entered into between-subjects analyses, whereas dimension weights and variance in average similarity ratings could be computed for each individual.

\section{Hypothesis 1: External Validity of Racial Essentialism and Perceived Race Scale}

Because the perceived race scale was an ad hoc measure created to gauge how Black, White, or multiracial participants perceived a face to be, I used linear regression to predict the ratings of perceived race by racial essentialism with the expectation that racial essentialism would predict lower ratings of Black and ambiguous faces, corresponding to perceptions of those faces as being more "Black". This would serve to provide external validity to the Race Conceptions Scale in confirming that it was measuring a construct which predicts more categorical views of race and hypodescent, as well as provide some validity to the ad hoc measure in that it captured the construct of perceived race. Racial essentialism significantly predicted the ratings of Black faces $\left(\beta=-.17, p=.04, R^{2}=.03\right)$, where higher racial essentialism resulted in lower scores on a scale from $0=$ Black to $100=$ White, but this effect only accounted for 3 percent of the variance in the ratings. Racial essentialism did not predict the ratings of White $(\beta=.10, p=.22)$ or Ambiguous faces $(\beta=-.02, p=.81)$, nor did it predict the variation in ratings within the face types ( $p$ 's from .35 to .97 ). This finding partially supports Hypothesis 1 in that higher racial essentialism predicted rating Black target faces more categorically (close to 0), but it did not predict the hypodescent of Ambiguous faces as expected from racial essentialism literature.

\section{Race as a Defining Dimension in Facial Perception: nMDS Analyses}

In conducing the multidimensional scaling analyses, I initially scaled the matrices from two to six dimensions. To determine the best dimensionality of each solution, I followed the procedure suggested by Fitzgerald and Hesson-McInnis (1989) whereby the stress values were evaluated by comparing each to 
past stress values obtained through Monte Carlo simulations of randomly generated data from Klahr (1969). Stress in nMDS represents a badness-of-fit index, where higher values indicate worse fit (Borg \& Groenen, 2005). The criteria developed in Klahr (1969) were determined with a single aggregate matrix which, by nature, will produce lower stress values than if using individual differences scaling (INDSCAL), which requires multiple matrices that introduce more error variances (Carroll \& Chang, 1970). Consequently, for INDSCAL analyses with 4 or fewer matrices, I set the stress criteria at .10 or below, and for INSDCAL analyses with all participants (150+ matrices) I relied more on a multitude of factors: 1) the relative decrease in stress from a higher dimensional solution to a lower one similar to considering a scree plot, 2) the change in variance accounted for when comparing models $\left(R^{2}\right)$, and 3) the relative overall importance of each dimension, where a lower-dimensional solution would be preferred over a higher one if the extra dimensions present a fraction of the weights in comparison to the lower dimensions (Borg \& Groenen, 2005). An important note about INDSCAL that is different than standard multidimensional reduction with a single matrix is that the dimensional solution cannot be rotated, making it difficult to observe the same dimensions across different INSDCAL analyses.

\section{Hypothesis 2: Common Race Dimension in All Solutions}

To examine the cross-race effect between groups and within participants, I scaled two different sets of participant matrices. The first used the aggregate mean ratings of the secure and neutral prime groups to compare the Euclidean distances between groups which was scaled to three dimensions (referred to henceforth as the Group solution), and the second used all 150 participant matrices to compare the individual differences in dimension weights which was scaled to four dimensions (Individual solution). I regressed the perceived race ratings on the nMDS coordinates of the faces in the first dimension for both solutions to verify that the first dimension corresponded to a race dimension as hypothesized (Hout et al., 2013). For both the Group and Individual solutions, the first dimension was strongly and significantly predicted by the participant's ratings of perceived race for the 20 faces $(\beta=-$ $.99, p<.001)$. The nature of the dimensions was then probed by comparing relative dimension weights. Weights represent the importance of a dimension in determining the distances between stimuli in the 
group- or individual-level dimensional solution, ranging from 0 to 1 . The first dimension in the 3dimensional Group solution was the most important overall, with a weight of .85 in comparison to weights of .05 and .04 in the second and third dimensions. For the Individual solution, the race dimension was just over twice as important as the other three dimensions with a weight of .13. Dimension 2 had a weight of .06 , dimension 3 of .05 , and dimension 4 of .05 . Visual scanning of face stimuli on the first dimension suggests that this dimension was sorted by lightness of skin tone (Figure 2). This result supported Hypothesis 2 that race would appear as the most important dimension in the perception of faces. No other dimensions were significantly predicted by the perceived slider task ratings.

\section{Hypothesis 3a.1 - 3a.2: Security's Impact on Perceptual Structures}

I evaluated the difference in the cross-race effect between groups by generating separate facespaces for the security priming group and the neutral priming group. This was accomplished by scaling the 76 secure participant matrices separately from the 74 neutral participant matrices, which were both scaled in 4 dimensions. The average distances and standard deviations for the three face types in each prime group is provided in Table 1. There were significant differences in the distances between the three face types for participants regardless of prime condition $(F[2,110]=13.28, p<.001)$, where the Black faces had significantly smaller distances than the White and Ambiguous faces (Helmert contrast $=-0.90$, $p<.001,95 \% \mathrm{CI}=[-1.27,-0.53])$, but the White and Ambiguous faces were not significantly different (Helmert contrast $=-0.18, p=.37,95 \% \mathrm{CI}=[-.56, .21])$. 
Table 1

Crosstabulation of Average Euclidean Distances Within Face Types by Prime Group

\begin{tabular}{lllllll}
\hline & \multicolumn{2}{c}{ Black } & \multicolumn{2}{c}{ Ambiguous } & \multicolumn{2}{c}{ White } \\
\cline { 2 - 7 } Prime & Mean & $S D$ & Mean & $S D$ & Mean & $S D$ \\
\hline Secure & 1.44 & .85 & 2.43 & .88 & 2.27 & .82 \\
Neutral & 1.42 & .92 & 2.42 & .89 & 2.23 & .78 \\
\hline
\end{tabular}

Note. Presents the means and standard deviations of the Euclidean distances within same-race stimuli in the 3-dimensional group-level nMDS solution. Black, Ambiguous, and White refer to the race of the target stimuli.

\section{Figure 2}

Comparison of Faces Distributed on Dimension 1 and the Perceived Race Scale

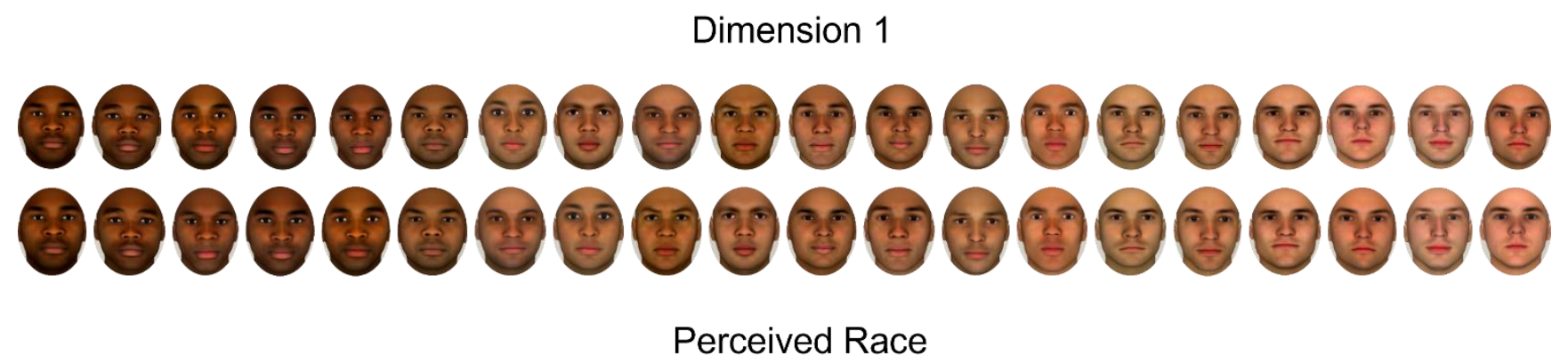

Note. The largest discrepancies between dimension 1 and average perceived race occurred amid the Ambiguous faces. Black and White faces had only one position switched.

In comparing the Euclidean distances between same-race faces for the participants in security priming versus neutral priming groups, there was no main effect of prime group on face-pair distances, nor was there a significant interaction of prime group and face race $\left(F_{\text {prime }}[1,110]=.02, p=.90\right.$; $\left.F_{P x R}[2,110]<.01, p>.99\right)$. Hypothesis $3 a .1$ was not supported in that the security prime did not impact the emergence of the cross-race effect in Euclidean distances between same-race faces.

To compare the category judgements of the Black, White, and ambiguous-race faces between the securely primed and neutrally primed participants (Hypothesis 3a.2), I aggregated the similarity ratings of 
the two prime groups into separate $20 \times 20$ matrices which represent the average similarity judgements between all possible face pairs. These two matrices were scaled using INDSCAL in two to six dimensions. The three-dimensional scaling was chosen as a best option due to its balance of low-stress (.10) and variance captured $\left(R^{2}=.94\right)$. The four-dimensional solution captured only $1 \%$ more variance with a stress value of .08. There was only a small observed difference in the weight of the race dimension from the aggregate secure prime matrix (.922) and the aggregate neutral prime matrix (.917), potentially suggesting that the priming effect did not decrease reliance on the race dimension in similarly ratings as expected (Hypothesis 3a.2).

\section{Individual Differences Scaling for All Participants}

Because the multidimensional scaling analysis did not allow for significance testing for the difference between these weights, I conducted an additional INDSCAL analysis with all 150 participant matrices to generate dimension weights for each participant that could be used in a MANCOVA. It should be noted that this resulted in a different dimension reduction that would not be guaranteed to contain the same dimensions as before.

In this case, the four-dimensional solution showed the greatest trade-off of stress (.24) to dimension interpretability and captured $30 \%$ of the variance in participant similarity ratings (Figure 3). In this solution, mean Euclidean distance for Black faces was $.84(S D=0.53)$, for White faces was 2.28 ( $S D$ $=0.72)$, and for Ambiguous faces was $2.21(S D=1.00)$. A one-way ANOVA revealed significant differences in the Euclidean distance based on the race of the face pairs, $F(2,55)=6.90, p=.002$. Post hoc Scheffe comparisons showed significant differences between the Black face pair distances and both the White $\left(M_{\mathrm{D}}=-0.83, p=.03,95 \%\right.$ CI: $\left.[-1.61,-.06]\right)$ and Ambiguous $\left(M_{\mathrm{D}}=-0.98, p=.003,95 \%\right.$ CI: [1.66, -.30]) face pair distances, $p<.001$. The difference between the White and Ambiguous face pair distances was not significant, $M_{\mathrm{D}}=--0.14, p=.86,95 \% \mathrm{CI}:(-.83, .53)$. Taken together, this result validates that the cross-race effect was captured in the nMDS solution considered in the subsequent analyses. 


\section{Figure 3}

\section{Four-Dimensional Individual Differences Model Generated with All Participant Matrices}

A.

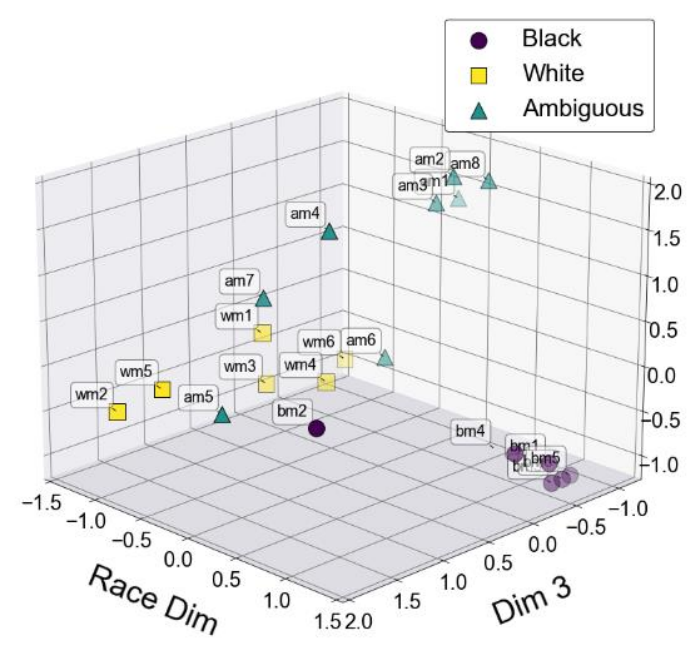

B.

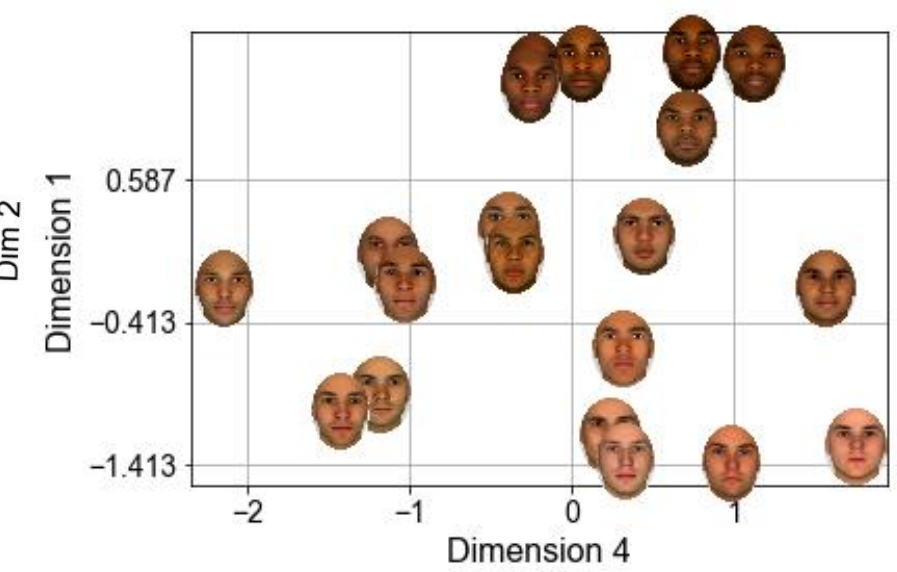

Note. A. Three-dimensional depiction of the four-dimensional solution of similarity between pairs of 20 faces; "am" refers to ambiguous targets, "bm" refers to Black targets, and "wm" refers to White targets. $B$. Fourth dimension plotted two-dimensionally with the first dimension.

\section{Security Priming Effect Testing: MANOVAs}

A series of MANOVAs were conducted to examine security priming's ability to decrease the cross-race effect in terms of average similarity ratings and the variation in ratings, and to impact the distribution of dimension weights. Before conducting them, I tested group homogeneity in the two priming conditions on relevant dimensions. A chi-square test of homogeneity assessing the distribution of ethnicities in the two priming conditions revealed no significant departures from the expected cell sizes, indicating that random assignment worked as intended, $\chi^{2}(3, \mathrm{~N}=147)=1.55, p=.67$ (Table 2). In addition, a Mann-Whitney $\mathrm{U}$ test revealed no significant differences in reported contact with Black friends, teachers or mentors between the two prime groups, $U\left(N_{\text {Neutral }}=75, N_{\text {Secure }}=75\right)=2750.00, Z=-.24$, $p=.81$. However, there were significant differences in reported contact with white friends, teachers, and mentors between the two priming groups, $\left(U\left[N_{\text {Neutral }}=72, N_{\text {Secure }}=74\right]=2048.00, z=-2.54, p=.01\right)$, where 
the secure group reported lower contact with white individuals on average than the Neutral group. This, however, had a small effect size of .04 and was determined negligible. Lastly, there were no significant differences in ingroup identification with Black identity $(t[140]=.00, p>.99)$ or white identity $(t[140]=$ $-.53, p=.60)$ between groups.

\section{Table 2}

Cell Sizes of Racial-Ethnic Identities by Prime Group, Overall and Included in Analyses

\begin{tabular}{llll|lll}
\hline & \multicolumn{3}{c|}{ Overall Total } & \multicolumn{3}{c}{ Analysis Total } \\
\cline { 2 - 7 } Ethnicity & Secure & Neutral & Total & Secure & Neutral & Total \\
\hline White & 58 & 50 & 108 & 47 & 54 & 101 \\
Black & 4 & 7 & 11 & 6 & 4 & 10 \\
Asian & 4 & 4 & 8 & 3 & 3 & 6 \\
Hisp/LatX & 9 & 11 & 20 & 10 & 9 & 19 \\
\hline Total & 72 & 75 & $N=147$ & 66 & 70 & $N=136$ \\
\hline
\end{tabular}

Note. Total sample size $(N)$ was 150 , with $n=8$ participants not disclosing Racial-Ethnic identity.

Attrition from overall to analysis is due to 1) participants not completing both the similarity ratings and the perceived race ratings, and 2) exclusion criteria such as taking 7 or more days between Sessions 1 and $2(n=7)$. 


\section{Factors Impacting the Prime Effect}

After the explicit prime in session one, neutral-primed participants reported an average Felt Security of $74.68(\mathrm{SD}=19.31)$ and secure-primed participants reported an average of $96.66(\mathrm{SD}=15.24)$. There was a highly significant difference in reported feelings of Felt Security between the groups, $t(142.60)=-7.84, p<.001$, Cohen's $d=1.26$, suggesting security priming manipulation was effective as intended. In determining whether attachment styles interacted with the security prime, a one-way analysis of variance (ANOVA) with continuous measures of Anxiety and Avoidance as covariates revealed that, separately, anxiety and avoidance did not significantly interact with the prime $\left(F_{\mathrm{A}}[1,151]=1.63, p=.20\right.$, $\eta_{\mathrm{p}}^{2}=.01$ for attachment anxiety; $F_{\mathrm{AV}}[1,151]=3.39, p=.07, \eta_{\mathrm{p}}{ }^{2}=.02$ for attachment avoidance). Total ECR scores representing insecurity (where low scores [9] indicate security and high scores [63] indicate a combination of high attachment anxiety and avoidance) did significantly impact felt security depending on prime group, where the security prime group experienced a decrease in felt security by -0.16 units per increase in 1 unit of their combined anxious/avoidant attachment score, $F(1,152)=5.78, p=.02, \eta_{\mathrm{p}}{ }^{2}=$ $.04, \Delta R^{2}=.03$. This suggested that, in those with trait-level attachment insecurity, security priming slightly decreased felt security.

\section{Hypothesis 3a.3: Security Effects on Dimension Weights and Similarity Ratings}

Before testing the impact of the prime on dimension weights and similarity ratings, I excluded participants who took 7 days or more between the first and second prime $(n=7)$, leaving 143 participants for analysis. A one-way MANOVA was conducted with dimension weights and average similarity ratings between same-race faces entered as dependent variables, priming condition as the independent variable, and racial essentialism as a covariate. Dimension weights and average similarity ratings between samerace faces constituted two indicators of the cross-race effect. Hypothesis $3 a .3$ was not supported, as the model did not account for a significant proportion of variance for any of the dependent variables, and neither prime group nor racial essentialism contributed to the four-dimension weights or to the same-race face similarity ratings (Wilks' $\Lambda_{\text {prime }}=.98, p=.18$; Wilks' $\Lambda_{\mathrm{RE}}=.92, p=.67$ ). 


\section{Hypothesis 4: Change in Racial Essentialism}

An independent samples t-test assessing the difference between racial essentialism at time one and time two between groups revealed that the average change in racial essentialism for both priming groups $\left(M_{\text {DNeutral }}=-2.03, S D=7.90, n=68 ; M_{\text {DSecure }}=-.82, n=73, S D=7.98\right)$ was not significantly different, $t(139)=-.90, p=.37$, Cohen's $d=.15$. Contrary to the Hypothesis 4 , the decrease in racial essentialism in the Neutral prime group was significant with a small effect size $\left(t_{\text {Neutral }}[67]=-2.12, p=\right.$ .04 , Cohen's $d=.12$ ), whereas the decrease in racial essentialism in the secure group was not significant, $t_{\text {Secure }}(72)=-.88, p=.38$, Cohen's $d=.05$. Thus, Hypothesis 4 which predicted security priming would decrease racial essentialism was not supported.

\section{Hypothesis 5: Prime, Racial Essentialism, and Perceived Race}

The average perceived race ratings and standard deviations for Black, Ambiguous, and White faces are presented in Table 3. To test whether a security prime impacted hypodescent of ambiguous faces, I performed a one-way MANCOVA with prime group predicting the average race ratings of Black, Ambiguous, and White faces, as well as the variation in those ratings for each face type, while including average racial essentialism as a covariate. There were no significant multivariate effects of average racial essentialism (Wilks' $\Lambda=.96, p=.42, \eta_{p}{ }^{2}=.04$, power $=.39$ ) or prime group (Wilks' $\Lambda=.94, p=.25, \eta_{p}{ }^{2}$ $=.06$, power $=.51)$ on the ratings of perceived race for the 20 faces, nor on the variation in ratings of the Black, White, and Ambiguous faces. Taken together, the security priming effect was not observed decreasing the tendency for hypodescent when the level of racial essentialism was controlled. 
Table 3

Average Ratings of Perceived Race for Black, Ambiguous, and White Faces

\begin{tabular}{llllllll}
\hline \multirow{2}{*}{ Prime } & \multicolumn{2}{c}{ Black } & \multicolumn{2}{c}{ Ambiguous } & \multicolumn{2}{c}{ White } \\
\cline { 3 - 7 } & Ethnicity & Mean & $S D$ & Mean & $S D$ & Mean & $S D$ \\
\hline Secure & Total & 9.47 & 8.60 & 48.27 & 8.59 & 85.92 & 8.76 \\
& White & 10.62 & 8.95 & 48.63 & 9.02 & 85.78 & 8.61 \\
& Non-White & 5.78 & 6.30 & 47.12 & 7.18 & 86.35 & 9.51 \\
\hline Neutral & Total & 11.88 & 9.03 & 51.60 & 9.49 & 85.84 & 9.70 \\
& White & 11.32 & 9.54 & 52.16 & 10.26 & 87.17 & 9.64 \\
& Non-White & 13.31 & 7.64 & 50.17 & 7.23 & 82.43 & 9.26
\end{tabular}

Note. On a 0 (Black) to 50 (Multiracial) to 100 (White) scale. The racial categories of Black,

Hispanic/LatinX and Asian have been collapsed into the category "non-white." White $n=91$, non-white $n$ $=34$. 


\section{Supplementary Analyses: A Moderating Role of Ingroup Identification and Ethnicity}

The main study hypothesis that security priming effect would decrease perception-level outgroup bias (i.e., cross-race effect) was not supported. To explore potential reasons, I examined participant ingroup identification and ethnicity as potential moderators. Past literature has found consistently effective moderators of the cross-race effect to be social context and the perception of ingroups and outgroups (Hehman, Mania, \& Gaertner, 2010; Marsh, Pezdek, \& Ozery, 2016), and specifically the initial perception of racial ingroups (Dickter \& Bartholow, 2007; Shriver, Young \& Hugenberg, 2007).

Unfortunately, the sample was majority white, and any analyses performed considering a fourlevel factor of ethnicity would be too low-powered (some subsample sizes would be as low as 2). A binary variable was instead computed to represent white/non-white status, with 103 participants as white and 34 as non-white (8 did not report an ethnicity). The subsample sizes using white/non-white status were still underpowered (15 and 18 in the neutral and secure groups), so any interactions are to be interpreted with care (Table 4).

Participants reported two different measures of ingroup identity, but likely because most participants were white, the measure of Black ingroup identity was not a sufficient covariate in any of the supplementary models because of its low mean and standard deviation $(M=1.91, S D=1.73)$. Therefore, only white ingroup identification was considered as a measure of ingroup identification in further analysis. 


\section{Table 4}

Cell Sizes of White/Non-White Status by Prime Group

\begin{tabular}{llll|lll}
\hline \multirow{2}{*}{ Ethnicity } & \multicolumn{3}{c|}{ Similarity Ratings } & \multicolumn{3}{c}{ Perceived Race } \\
\cline { 2 - 7 } & Secure & Neutral & Total & Secure & Neutral & Total \\
\hline White & 51 & 45 & 96 & 51 & 46 & 98 \\
Non-white & 15 & 18 & 33 & 16 & 18 & 35 \\
\hline Total & 66 & 63 & $N=129$ & 67 & 64 & $N=131$
\end{tabular}

Note. Cell sizes for similarity ratings and perceived race analyses. The racial categories of Black, Hispanic/LatinX and Asian have been collapsed into the category "non-white."

\section{White Ingroup Identification and Ethnicity Moderating Cross-Race Effect}

I conducted a $2 \times 2$ MANOVA with dimension weights and similarity ratings between same-race faces as dependent variables (i.e., same as hypothesis 3 testing), white ingroup identification as a covariate, and white/non-white status as another factor (i.e., in addition to priming groups) to probe for potential interactions. There were significant multivariate effects from white ingroup identification (Wilks' $\Lambda=.89, p=.03, \eta_{\mathrm{p}}{ }^{2}=.12$ power $=.84$ ) and from the interaction of white/non-white status and prime group (Wilks' $\Lambda=.85, p=.01, \eta_{\mathrm{p}}^{2}=.15$, power $=.93$ ) on the dimension weights and similarity ratings. Racial essentialism did not significantly impact any dimension weights or similarity ratings (Wilks' $\Lambda=.91, p=.12, \eta_{\mathrm{p}}{ }^{2}=.09$, power $=.67$ ), and there was no main effect of white/non-white status (Wilks' $\Lambda=.93, p=.29, \eta_{\mathrm{p}}^{2}=.07$, power $\left.=.51\right)$ or prime group $\left(\right.$ Wilks' $\Lambda=.94, p=.36, \eta_{\mathrm{p}}{ }^{2}=.06$, power $=.46)$.

In analyzing the between-subjects effects, it was revealed that white ingroup identification significantly impacted average same-race similarity ratings for all three face types, where higher white ingroup identification was associated with higher similarity ratings for all face types (Table 5). 
Table 5

Between-Subjects Effects on Dimension Weights and Face Similarity Ratings

\begin{tabular}{|c|c|c|c|c|c|}
\hline Predictor & Variable & $F$ & $p$ & $\eta_{\mathrm{p}}^{2}$ & Obs. Power \\
\hline \multirow[t]{7}{*}{ White Ingroup ID } & Dim1 & 1.07 & .30 & .01 & .18 \\
\hline & Dim2 & 2.89 & .09 & .02 & .39 \\
\hline & Dim3 & 3.46 & .07 & .03 & .45 \\
\hline & Dim4 & 1.94 & .17 & .02 & .28 \\
\hline & $\mathrm{B}-\mathrm{B}$ rating & 9.12 & $.003 * *$ & .07 & .85 \\
\hline & A-A rating & 6.61 & $.01 * *$ & .05 & .72 \\
\hline & $\mathrm{W}-\mathrm{W}$ rating & 5.41 & $.02 * *$ & .04 & .64 \\
\hline \multirow[t]{7}{*}{ Racial Essentialism } & Dim1 & 0.06 & .81 & $<.001$ & .06 \\
\hline & Dim2 & 1.25 & .27 & .01 & .20 \\
\hline & Dim3 & 0.55 & .46 & $<.001$ & .11 \\
\hline & Dim4 & 0.93 & .34 & .01 & .16 \\
\hline & $\mathrm{B}-\mathrm{B}$ rating & 0.18 & .67 & $<.001$ & .07 \\
\hline & A-A rating & 3.29 & .07 & .03 & .44 \\
\hline & $\mathrm{W}-\mathrm{W}$ rating & 0.33 & .57 & $<.001$ & .09 \\
\hline \multirow[t]{7}{*}{ White/NW } & Dim1 & 0.03 & .86 & $<.001$ & .05 \\
\hline & Dim2 & 1.05 & .31 & .01 & .17 \\
\hline & Dim3 & 2.40 & .12 & .02 & .34 \\
\hline & Dim4 & 2.72 & .10 & .02 & .37 \\
\hline & B-B rating & 1.36 & .25 & .01 & .21 \\
\hline & A-A rating & 0.37 & .55 & $<.001$ & .09 \\
\hline & $\mathrm{W}-\mathrm{W}$ rating & 0.83 & .36 & .01 & .15 \\
\hline \multirow[t]{7}{*}{ Prime } & Dim1 & 2.04 & .16 & .02 & .29 \\
\hline & Dim2 & 5.21 & $.02 *$ & .04 & .62 \\
\hline & Dim3 & 3.39 & .07 & .03 & .45 \\
\hline & Dim4 & 3.48 & .06 & .03 & .46 \\
\hline & B-B rating & 0.74 & .39 & .01 & .14 \\
\hline & A-A rating & 0.12 & .73 & $<.001$ & .06 \\
\hline & $\mathrm{W}-\mathrm{W}$ rating & 0.01 & .94 & $<.001$ & .05 \\
\hline
\end{tabular}




\begin{tabular}{llllll}
\hline Predictor & Variable & $F$ & $p$ & $\eta_{\mathrm{p}}^{2}$ & Obs. Power \\
\hline Prime*White/NW & Dim1 & 6.58 & $.01^{*}$ & .05 &. $\mathbf{7 2}$ \\
& Dim2 & 10.10 & $.002^{* *}$ & .07 & .88 \\
& Dim3 & 4.62 & $.03^{*}$ & .04 & .57 \\
& Dim4 & 6.66 & $.01^{*}$ & .05 & .73 \\
& B-B rating & 0.17 & .68 & $<.001$ & .07 \\
& A-A rating & 4.16 & $.04^{*}$ & .03 & .53 \\
& W-W rating & 1.00 & .32 & .01 & .17
\end{tabular}

Note. White Ingroup ID = white ingroup identification; Racial Essentialism = average racial essentialism score; White/NW = white/non-white status; Prime = secure or neutral prime group; Prime*White/NW = interaction of prime group and white/non-white status. "Dim1" - "Dim4" refer to dimensions 1 through 4. "B-B" refers to the average similarity rating of Black face pairs, "A-A" to ambiguous face pairs, and "WW" to White face pairs. Degrees of freedom $=1,125$.

$* p<.05, * * p<.01, * * * p<.001$

To tease apart the effect of white ingroup identification on same-race similarity ratings further, I conducted a test of moderation with Hayes' PROCESS macro using a simple moderation model (model 2) where prime group moderated the effect of white ingroup identification on similarity ratings of Black face pairs. When analyzed with white participants only $(N=97)$, the interaction was only marginally significant, $t(1,93)=1.72, p=.09$. When Ambiguous similarity ratings were entered as a predictor, the interaction of prime group and white ingroup identification was significant $\left(F[1,93]=4.12, p=.05, \Delta R^{2}\right.$ $=.04$ ), with the secure prime group having significantly higher similarity ratings between ambiguous-race faces when white ingroup identification was higher. Because this was the opposite of the expected effect, I included felt security as a mediator to test whether it was through heightened feelings of security that the similarity ratings increased with higher white ingroup identification (model 5 in PROCESS), or if the security prime unintentionally primed a different feeling in the white participants. This model was significant $(F[4,92]=4.74, p=.002)$, with prime group, felt security, and the interaction of prime group and white ingroup identity having significant direct effects on average ambiguous face similarity ratings 
(Table 6). Felt security additionally had a significant indirect effect on average ambiguous face similarity ratings $(B=.28,95 \% \mathrm{CI}:[.04, .57])$, confirming that it was through increased feelings of security that the prime was impacting average similarity ratings.

The interaction between prime group and white/non-white status significantly impacted the weights for dimensions 1,2 , and 4 with good power (.72 to .88), however, other significant effects such as the one on dimension 3 and the Ambiguous face similarity ratings were low in power due to the small subsample sizes. The direction of interactions with the four dimension weights would suggest that the security prime impacted non-white participants differently than white participants (Figure 4). Securely primed non-white participants used slightly different category judgements than neutrally primed, nonwhite participants as well as white participants in both prime conditions: securely primed, non-white participant dimension weights were chronically lower for all four dimensions.

\section{Table 6}

Ambiguous Similarity Ratings Predicted by White Ingroup Identification, Prime \& Felt Security

\begin{tabular}{llllll}
\hline Predictor & $B$ & $S E$ & $t$ & $p$ & $R^{2}$ \\
\hline White Ingroup & .05 & .08 & 0.57 & .57 & .17 \\
Prime & -1.79 & .83 & -2.16 & $.03^{*}$ & \\
Felt Security & .01 & .01 & 2.30 & $.02^{*}$ & \\
Prime*White Ingroup & .29 & .13 & 2.23 & $.03^{*}$ & \\
\hline
\end{tabular}

Note. The model included direct effects of white ingroup identification, prime group, felt security, and the interaction of prime group and white ingroup identification on average similarity ratings of ambiguous faces, with an additional indirect effect of white ingroup identification through the mediator of felt security. Prime group was entered as a moderator only for the direct effect of white ingroup identification. $* p<.05$ 


\section{Figure 4}

Interaction of Prime Group and White/Non-White Status Across the Four Dimensions
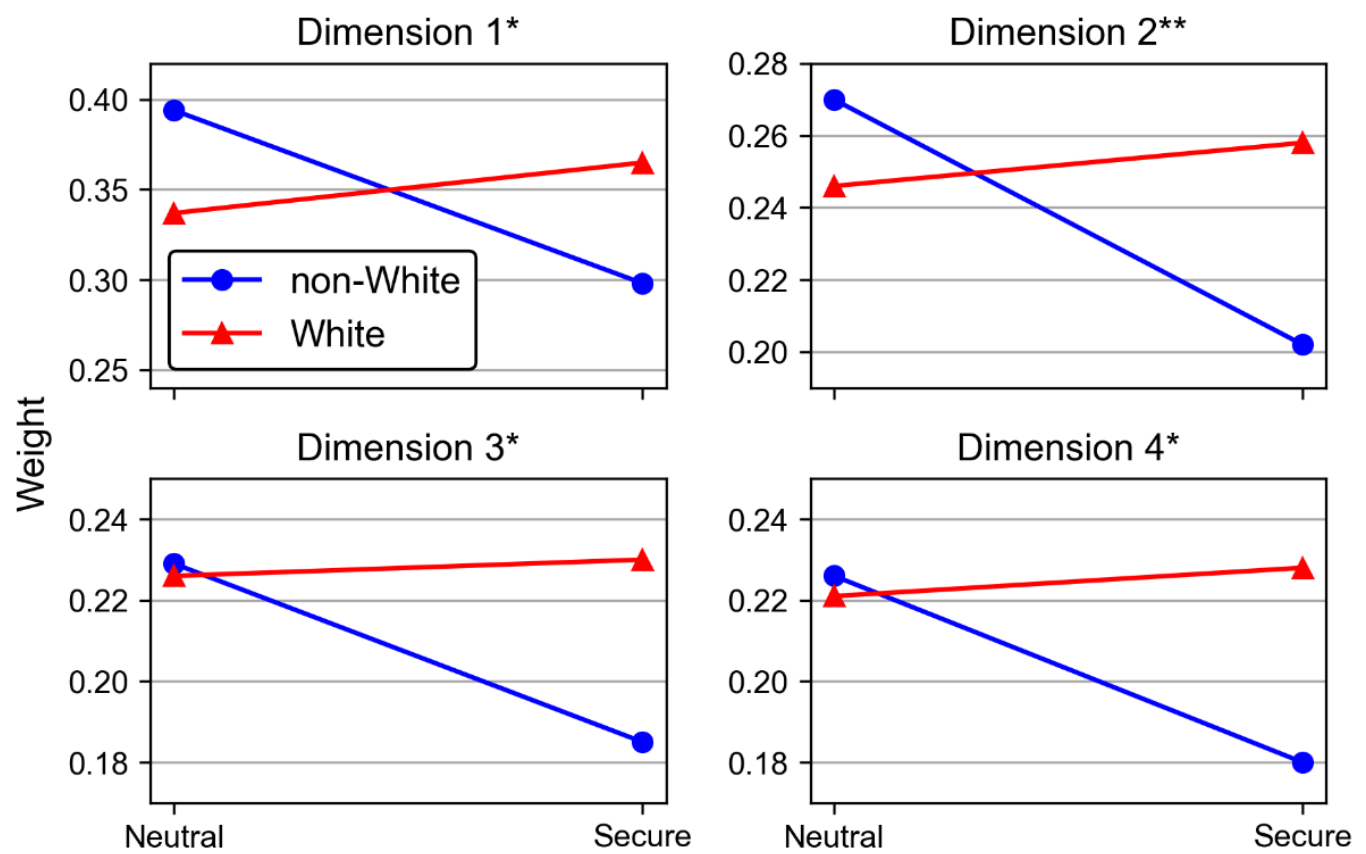

Note. Estimated marginal means were evaluated with the covariates of white ingroup identification set at 5.40 and racial essentialism set at 89.10. Interactions depict non-white participants in the secure prime group had lower usage of all dimensions, indicating the security prime did impact dimension weights but only for non-white participants.

$* p<.05$

In addition to being a strong covariate for similarity ratings, white ingroup identification had strong multivariate effects on the variation in similarity ratings of same-race faces, but racial essentialism, white/non-white status, and prime group did not (Table 7). White ingroup identification highly significantly impacted the variation in similarity ratings between Black faces where higher white ingroup identification lowered the variation between Black similarity ratings $F(1,125)=12.44, p<.001, \eta_{\mathrm{p}}{ }^{2}=.09$, power $=.94$. There was a marginally significant effect of white ingroup identification on the variation in ambiguous face ratings $\left(F[1,125]=3.70, p=.06, \eta_{\mathrm{p}}{ }^{2}=.03\right.$, power $\left.=.48\right)$, but no significant effect on the variation in white face ratings, $F(1,125)=1.92, p=.17, \eta_{\mathrm{p}}^{2}=.02$, power $=.28$. 
I again tested the effect of white ingroup identification on the variation in Black face pair similarity ratings with prime group as a moderator, and found only a marginally significant interaction, $B$ $=-.13, t(1,93)=-1.74, p=.08$. White ingroup identification was also not significantly moderated by prime group when predicting the variation in Ambiguous similarity ratings, $B=.02, t(1,93)=.25, p=$ .80. Figure 5 displays the moderation interactions for both average similarity ratings and standard deviations of Ambiguous face pairs.

\section{Table 7}

Multivariate Effects on the Variation in Same-Race Similarity Ratings

\begin{tabular}{lllll}
\hline Predictor & Wilks' $\Lambda$ & $p$ & $\eta_{\mathrm{p}}{ }^{2}$ & Power \\
\hline White Ingroup & .91 & $.01^{* *}$ & .09 & .84 \\
Racial Ess. & .98 & .54 & .02 & .20 \\
White/Non-White & .99 & .90 & .01 & .08 \\
Prime Group & .98 & .53 & .02 & .21 \\
Prime*White/NW & .98 & .52 & .02 & .21 \\
\hline
\end{tabular}

Note. Effects of white ingroup identification, racial essentialism, prime group, and white/non-white status.

Degrees of freedom $=3,123$. Bold indicates acceptable power.

$* p<.05, * * p<.01, * * * p<.001$ 


\section{Figure 5}

White Ingroup and Similarity/Variation in Ambiguous Face Pair Ratings for White Participants

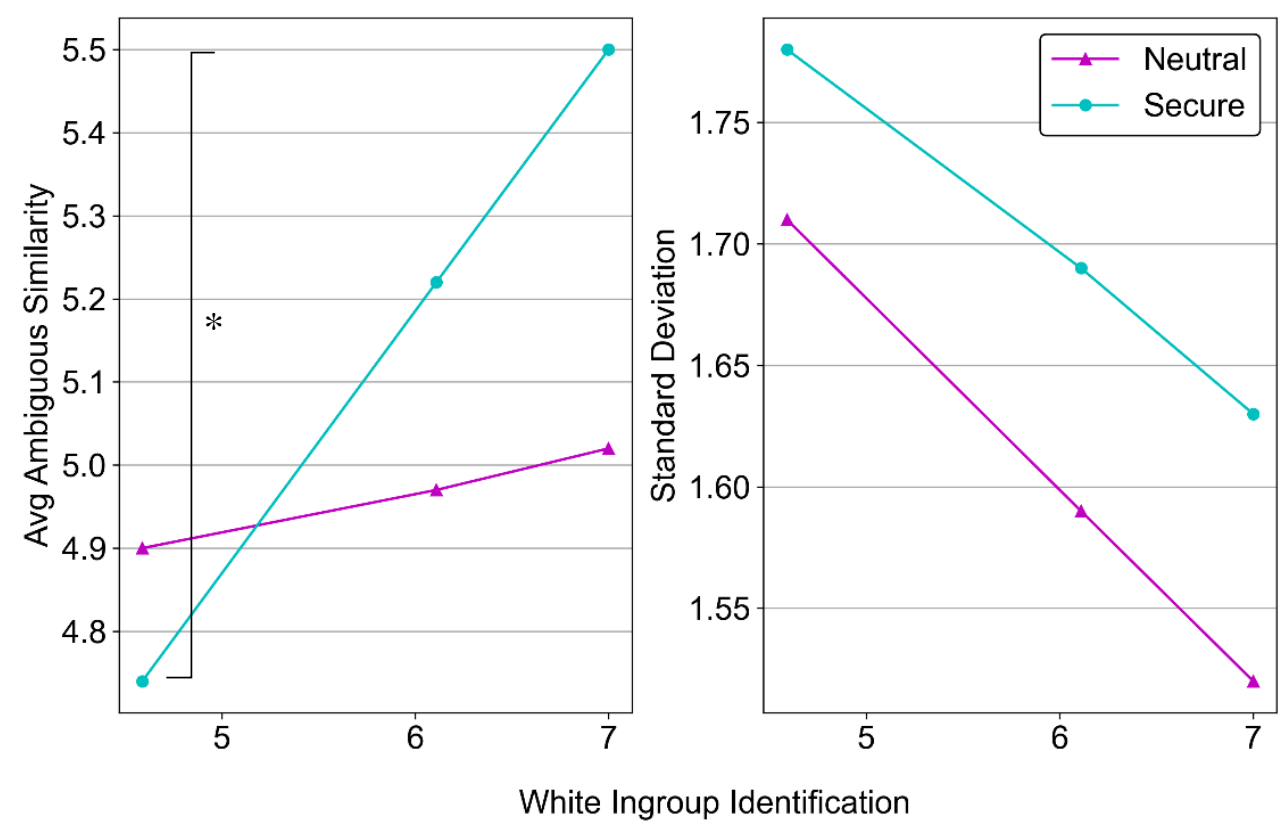

Note. Interactions represent $N=97$ white participants. Similarity scores could range from 1 (least similar) to 8 (most similar). Figure depicts that as identification with white identity increased, average similarity ratings for Ambiguous face pairs increased only for the securely primed participants. The decrease of variation in ratings was only marginally significant.

$* p<.05$

\section{Ethnicity Moderating Hypothesis 5: Effects of Secure Prime on Perceived Race}

To test the impact of white ingroup identification on perceived race ratings, I added it as a covariate to the original model where prime group and racial essentialism predicted the perceived race ratings and variation within the same-race ratings. I also added white/non-white status as another factor, conducting a 2 x 2 MANCOVA. White ingroup identification and racial essentialism were not significant covariates in the model, nor did white/non-white status have a main effect (Table 8).

However, prime group had significant multivariate effects, though this effect was qualified by a significant multivariate interaction effect of prime and white/non-white status. Table 9 displays the results 
of the between-subjects analysis. The effect of the secure prime significantly impacted average perceived race ratings for Black faces, where the secure group rated the Black faces as more Black $(M=9.46)$ than the neutral group $(M=11.88)$, but this effect was observed with low power (.62). This effect was also qualified by the interaction with ethnicity, where the effect is stronger for non-white participants, but this interaction was observed with low power as well (.63; Figure 6).

\section{Table 8}

Multivariate Effects on Perceived Race Ratings and Variation Within Same-Race Faces

\begin{tabular}{lcccc}
\hline Predictor & Wilks' $\Lambda$ & $p$ & $\eta_{\mathrm{p}}{ }^{2}$ & Power \\
\hline White Ingroup & .99 & .97 & .01 & .12 \\
Racial Ess. & .94 & .30 & .06 & .47 \\
White/non-white & .95 & .39 & .05 & .40 \\
Prime Group & .86 & $.01^{* *}$ & .14 &. $\mathbf{9 1}$ \\
Prime*White/NW & .88 & $.02^{*}$ & .12 & $\mathbf{. 8 5}$
\end{tabular}

Note. Effects of white ingroup identification, racial essentialism, prime group, and white/non-white status.

Degrees of freedom $=6,120$

$* p<.05, * * p<.01, * * * p<.001$ 


\section{Figure 6}

Black Faces: Prime Group and White/Non-White Status on Perceived Race Ratings

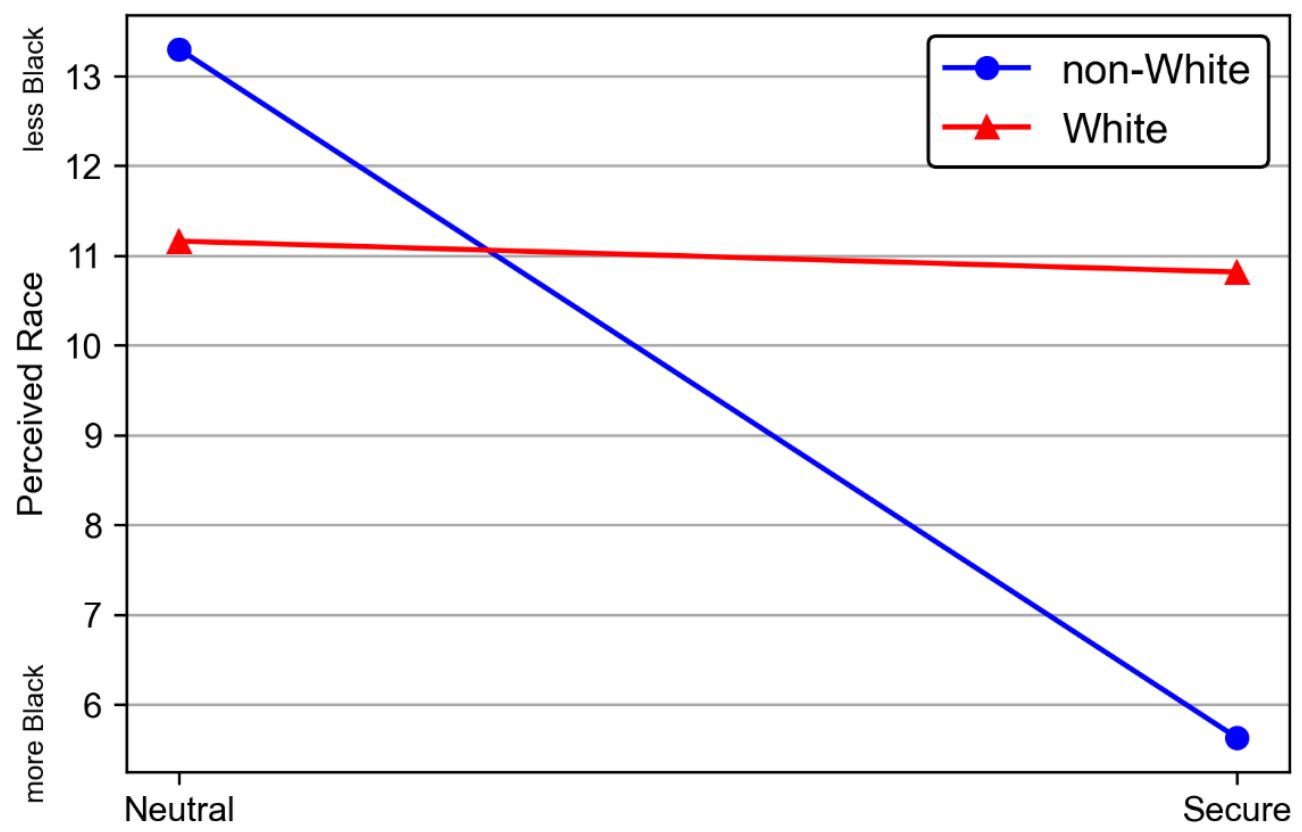

Note. Measured on a scale from 0 (Black) to 100 (White). There were no significant differences in the similarity ratings for Black faces in the neutral prime group, but non-white and securely primed participants rated Black faces as significantly "more Black” than white participants.

The prime effect also significantly impacted the variation in ratings of perceived race for ambiguous targets. However, this was qualified by a significant interaction effect between prime group and white/non-white status, where only the non-white and securely primed participants had significantly more variation in ambiguous face ratings $\left(M_{\text {Rating }}=47.12, S D=7.18 ; M_{\text {Variation }}=18.09, S D=8.26\right)$ compared to all other participants (Mean variations ranged from 10.89 to 13.36; Figure 7). To probe further, I calculated a variable to capture the trend in direction participants rated the Ambiguous faces. Mean differences in the non-white group ranged from -21.37 (about three-quarters Black) to 16.88 (about one-fifth white), with the overall average ratings of Ambiguous faces sitting just under the 50/50 mark ( $M$ $=-1.68, S D=8.44)$. These participants tended to utilize the full range of the scale rather than always rate 
Ambiguous faces in the middle and did not show a large tendency for hypodescent. There was no difference in the variation of ambiguous face ratings for white participants between the priming conditions.

\section{Table 9}

Between-Subjects Effects on Perceived Race and Variation in Race Ratings

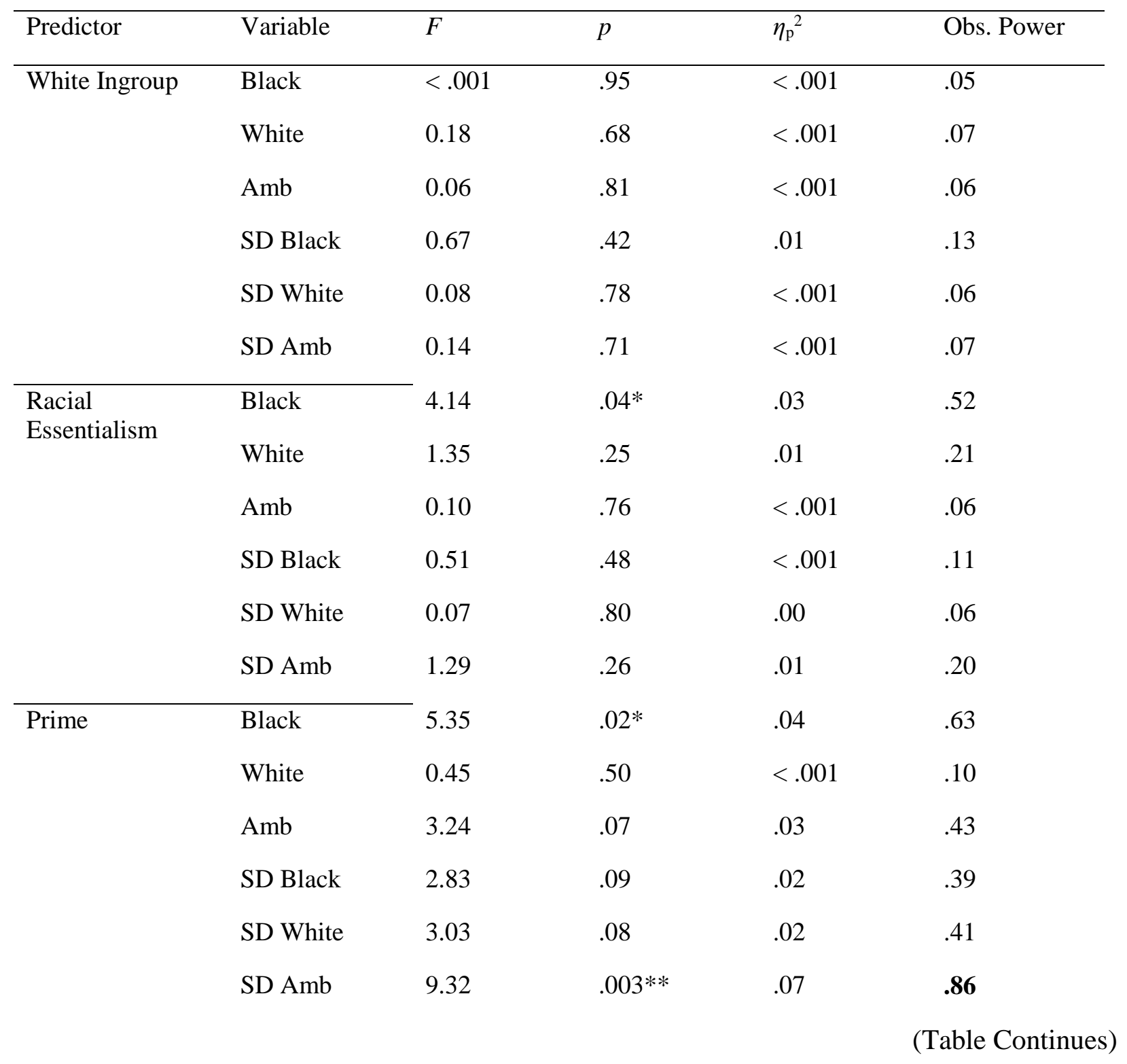




\begin{tabular}{llllll}
\hline Predictor & Variable & $F$ & $p$ & $\eta_{\mathrm{p}}{ }^{2}$ & Obs. Power \\
\hline White/NW & Black & 0.49 & .48 & $<.001$ & .11 \\
& White & 0.42 & .52 & $<.001$ & .10 \\
& Amb & 0.83 & .37 & .01 & .15 \\
& SD Black & 0.26 & .61 & $<.001$ & .08 \\
& SD White & 0.30 & .59 & $<.001$ & .08 \\
& SD Amb & 3.79 & .05 & .03 & .49 \\
& Black & 4.35 & $.04 *$ & .03 & .54 \\
& White & 2.47 & .12 & .02 & .34 \\
& Amb & 0.01 & .94 & $<.001$ & .05 \\
& SD Black & 0.97 & .33 & .01 & .16 \\
& SD White & 0.30 & .59 & $<.001$ & .08 \\
& SD Amb & 13.75 & $<.001 * * *$ & .10 & .96
\end{tabular}

Note. White Ingroup = white ingroup identification; Racial Essentialism = average racial essentialism score; White $/ \mathrm{NW}=$ white/non-white status; Prime $=$ secure or neutral prime group; Prime $*$ White $/ \mathrm{NW}=$ interaction of prime group and white/non-white status. Black, White and Amb. refer to the average perceived race rating participants made for that respective race category, and "SD" refers to the variation in similarity ratings as captured by standard deviation.

$* p<.05, * * p<.01, * * * p<.001$ 


\section{Figure 7}

Ambiguous Faces: Prime Group and White/Non-White status on Variation in Race Ratings

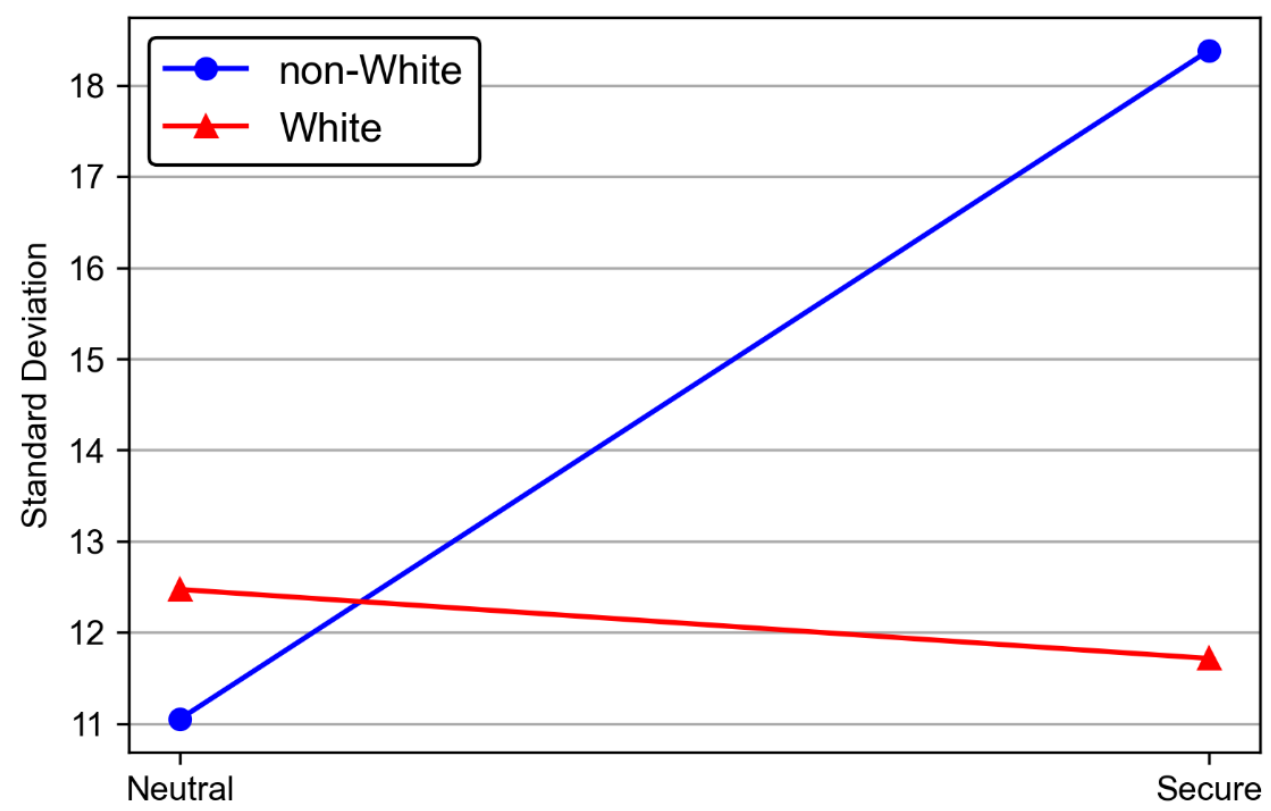

Note. Measured on a scale from 0 (Black) to 100 (White). Mean rating for neutral group = 51.60; Mean rating for secure group $=48.27$. There were no significant differences in the variation in race ratings for ambiguous faces in the neutral prime group, but non-white and securely primed participants had significantly more variability in their race ratings than white participants. Mean differences in the nonwhite group ranged from -21.37 (about three-quarters Black) to 16.88 (about one-fifth white), with the overall average ratings of Ambiguous faces sitting just under the 50/50 mark $(M=-1.68, S D=8.44)$. These participants tended to utilize the full range of the scale rather than always rate Ambiguous faces in the middle. 


\section{CHAPTER V: DISCUSSION}

Attachment security priming has been examined as an intervention to improve intergroup relations, emotional processing of threatening stimuli, and even temporarily increase cognitive ability (Mikulincer \& Shaver, 2001; Bai et al., 2019). Security priming's cognitive-attitudinal effects on the perception of outgroup members have been replicated in a multitude of studies, but its impact on perceptual-level phenomena regarding race and ingroup formation was not yet established. The purpose of this study was to examine how heightened feelings of attachment security would impact the way individuals perceive and categorize Black, White, and ambiguous-race faces, hypothesizing that the positive intergroup effects of the security prime would extend to the perception of outgroup faces. As a pervasive perceptual bias, the cross-race effect is likely the basis for the development of cognitiveattitudinal biases such as outgroup homogeneity and attribution error, as both of these relate to how one may perceive members of an outgroup race to look similar, act similarly, and ultimately attribute the actions of one member to all members (Stolier \& Freeman, 2016).

The first focus of the analysis was to determine to what extent outgroup homogeneity and ingroup heterogeneity biases exist at the perceptual level, and to test whether security priming and racial essentialism would impact the manifestation of these biases. The second focus was to determine whether a security prime would lessen the tendency for hypodescent of ambiguous-race faces, as hypodescent can feed into more problematic attitudes towards racial outgroup members (Williams \& Eberhardt, 2008).

\section{Interpretation of nMDS Dimensions}

The general face-space that was generated from the INSDCAL analysis of all 150 participant's similarity ratings was best represented in four dimensions. The first, and most important, dimension was a race dimension that appeared to be ordered by dark to light skin tones. Though participants were asked to rate how they perceived the race of each face, the near-congruent order of dimension one with the perceived race ratings indicates that the participants used skin tone as the main tool for judging race. The race dimension was just over twice as important as all other dimensions, and for the 3-dimensional solution with the aggregate secure and neutral group matrices, this race dimension accounted for nearly 
all the variance (84\%) in ratings. This was true for participants of all ethnicities. Additionally, the emergence of the cross-race effect where Black faces were clustered closer together than White faces was not only ubiquitous across different nMDS models (Group, Individual, and all participant solutions), it permeated all 4 of the dimensions analyzed in the all-participant solution, pointing to the severity of the processing bias. Together, these findings suggests that regardless of race or ethnicity, the perception of race and consequently the cross-race effect is a main component of our perceptions when tasked with comparing faces without any external social context, as found in Dickter and Bartholow's (2007) study on social context and face individuation. In addition, we tend to use skin tone as a main indicator of race and primarily base our similarity judgements off the difference in skin tone, validating Papesh and Goldinger's (2010) findings from a similar MDS investigation of Black and White face stimuli. Adults have been found to have greater sensitivity to both physiognomic and skin tone cues in racial cognition while children start with only skin tone as a cue (Dunham et al., 2015), but it seems that though adults can have greater physiognomic sensitivity, responding to facial feature cues may require more concentrated effort than using skin tone and are thus less heavily employed.

Analysis of the pattern of face distributions in the four dimensions also illuminated the relation between the cross-race effect and racial categorization, for, in this sample of largely white participants, the ambiguous faces were processed with a comparable amount of individuation as White faces. Examination of the 3D rotation of the 4-dimensions revealed that the White and Ambiguous faces had similar average distances but were spread along different dimensions. For white participants, this could mean that Ambiguous faces were not processed immediately as outgroup faces and were given the same processing resources as ingroup faces were. A possible explanation for this is that the Ambiguous faces required additional processing resources to establish whether they were an ingroup or outgroup, and thus allowed more detailed processing (Knowles \& Peng, 2005). Knowles and Peng (2005) found a similar result where the racially ambiguous faces were inspected significantly longer than monoracial faces as white ingroup identification increased. Another possible explanation is that the Black stimuli had much lower contrast in shading compared to the White and Ambiguous faces, making it more difficult to see the 
minute differences in features that were more easily seen in the lighter-toned stimuli. However, it cannot definitively be said that only the Black faces were subject to the cross-race effect (effectively making it a Black-race effect), for unlike in the White stimuli, there was a cluster of ambiguous faces that were heavily distinguished from Black and White faces which showed little individuation characteristic of the cross-race effect. This finding is a useful addition to the cross-race effect literature, as it is usually only White and Black target stimuli or ambiguous faces generated from a morph of just two faces that are being compared. In utilizing ambiguous faces capturing a larger variance in features, this study further contributes to the generalizability of the cross-race effect and how varying perceptions of race can impact it.

Speculation as to what the second, third, and fourth dimensions represent is based on the extremes of each dimension. The second dimension appears to represent ambiguity, with the Black and White faces loading lower and all but two Ambiguous faces loading higher. The two Ambiguous faces which load lower may have been perceived as White or Black, which makes sense as the Ambiguous stimuli were designed to be equally interpretable as Black or White (Pauker \& Ambady, 2009; Figure B1).

Dimension 3 appears to separate the less prototypical faces with distinctive features from the more prototypical ones, for example with the lighter-toned Black face and the olive-toned White face loading higher (Figure B2). The order of faces across dimension 3 loosely capture head shape but the order repeats within the prototypical and non-prototypical clusters.

To further interpret the second and third dimensions, Figure B3 displays these dimensions plotted in 2D space. Upon examination of the clusters, the faces load in four quadrants. The largest cluster (quadrant III) features all the prototypical and clearly White or Black face stimuli loading lowest in both dimensions. The second cluster (quadrant II), loading high in dimension 3 and low in dimension 2, features the non-prototypical but clearly White or Black faces. The third cluster (quadrant IV) includes the prototypical ambiguous faces, with the last two ambiguous faces loading in the first quadrant as nonprototypical ambiguous faces. The last quadrant (I) contains the faces which were perceived as 
ambiguous and not belonging clearly to the "Ambiguous" or "Multiracial" grouping established in quadrant IV. With dimension 3 plotted against dimension 2, it is clearer to see that, together, the two dimensions separate Black, Ambiguous, and White faces as they were perceived—some Ambiguous faces were truly ambiguous while others were grouped with White or Black faces. Only two of the eight Ambiguous stimuli were grouped as Black or White, suggesting that hypodescent is a phenomenon occurring at an attitudinal rather than perceptuo-cognitive level. From this study, we may conclude that without any context outside of facial features and skin tone, individuals are more easily able to create three racial category groupings from which to base their race cognitions.

The fourth dimension is difficult to interpret, but one important observation is that the lightertoned Black face is no longer separated from the other Black stimuli. Because there is no immediately clear pattern to the order of the stimuli, it is likely that this dimension involves physiognomic characteristics or other more abstract judgements, or that it is simply capturing noise in the 4-dimensional solution (Figure B4).

\section{Racial Essentialism and Perceptions of Race}

One of the main moderators considered in this study was racial essentialism, representing the extent to which one believes that race is biologically based and unchanging. Prior research has found racial essentialism to play a large role in the hypodescent of ambiguous-race faces (Ho, Roberts, \& Gelman, 2015), and there were mixed findings as to whether racial essentialism would improve or reduce ambiguous face processing. Likely due to the procedures used in past experiments testing hypodescent, individuals higher in racial essentialism tended to categorize ambiguous faces as Black when only given the categories of Black or White (e.g., Gaither et al., 2016). The findings from the present study highlight a different process: when given a continuous scale with three major category points of Black, multiracial, and White, participants tended to rate Ambiguous faces right in the center category of multiracial regardless of racial essentialism. This suggests that when free to make race judgements on a continuum, white participants will more easily use a "Multiracial" category rather than default to hypodescent. Sensitivity to this third racial group can also be observed in the spacing in the race dimension, where 
there are clear delineations between the White, Ambiguous, and Black stimuli. The differences in ratings arose at the Black endpoint of the scale, where racial essentialism predicted participants would rate these faces closer to zero, the categorical endpoint of the Black to White scale. Categorical views of race are consistent with the working definition of racial essentialism, though past research in hypodescent has not focused on the perceptions of Blackness in Black stimuli because hypodescent is a construct defined in terms of multiracial or ambiguous-race stimuli (Banks \& Eberhardt, 1998). The present study findings demonstrate another potential facet of hypodescent where, given the opportunity to rate Black stimuli on a continuum closer to multiracial, participants high in racial essentialism will eschew individuation in favor of using group membership alone to make race judgements.

In terms of racial essentialism's role in impacting perceptual structures of faces of different races, this study did not find that racial essentialism impacted how similar participants perceived two Black, White, or Ambiguous faces to be. The variation in the way participants categorized the faces was not significantly impacted by racial essentialism, even when accounting for white ingroup identification and ethnicity. It may be the case that racial essentialism, as an explicit attitude or belief, does not play as large a role in initial perceptions of race, but instead guides the more conscious decision-making processes when race is made more explicit, such as making a rating of how one perceives the race of a face as found in the current study. The nMDS procedure in this study was very intentional in not biasing participants to focus on race or any other kind of feature unequally, but to allow them to rate facial similarity in whatever way they saw fit. Without the explicit task goal to sort by race, it seems that racial essentialism did not have a strong impact on perceptual structures. However, it would be valuable for this to be confirmed in a separate study and determine if the effects of racial essentialism really are dependent on explicit task goals about race.

The last hypothesis regarding racial essentialism was that it would be mutable by a security prime due to security priming's ability to improve cognitive flexibility. Racial essentialism scores tended to decrease for all participants across sessions, but for those with heightened security the scores remained stable. Unlike previous interventions which successfully mitigated the racial rigidity stemming from 
racial essentialism (Slepian et al., 2014), the security prime did not affect racial essentialism as an explicit attitude or belief. Rather, there was the unexpected finding that the participants who received the neutral prime had significantly lower racial essentialism scores, while the securely primed participants had negligible differences between sessions. This difference could be explained in several ways. The first consideration is that the heightened feelings of security improved the participants' recall of their previous answers to the racial essentialism scale, leading to either conscious or subconscious biasing of their answers toward improving consistency across time. Supported by findings that security priming has been used in the past to improve aspects of memory (Bai et al., 2019; Mikulincer, Shaver \& Rom, 2013), this offers a plausible alternative explanation. Considering that the neutral prime group did see a significant decrease in racial essentialism at time 2, this would also mean that either the similarity rating task had an impact on racial essentialism that was mitigated by the security prime, or that RCS scores tend to decrease after subsequent administrations. The RCS had a test-retest reliability of .88 in this study, similar to the original study which had a slightly lower test-retest reliability of .82 after a month between administrations (Williams \& Eberhardt, 2008). This average test-retest reliability of .85 suggests that participant scores differ somewhat between administrations, but, unfortunately, Williams and Eberhardt did not report on whether scores tended to change in a specific direction at different time points. Based on this study, it appears that RCS scores tend to decrease after subsequent administrations likely due to practice effects.

The second consideration is that the neutral prime may have failed to be completely neutral, and that asking participants to think of an acquaintance from class lacked control in what kind of acquaintance they focused on and whether that acquaintance had a large emotional impact on them (e.g., the cute person who sat next to me; the person whom I argued with, etc.). This instruction may have primed participants to endorse a social cognitive frame of mind that appreciates and accepts diversity of dimensions that define individuals. 


\section{Security Priming and Outgroup Homogeneity}

Security priming has been found to impact the processing of emotional cues in faces during the same early stages of processing as ingroup-outgroup and racial categorization (Bai et al., 2019; Dickter \& Bartholow, 2007). In addition, those with trait secure attachment were found to have increased processing in brain areas associated with feature extraction (Tang et al., 2017). From this framework, I hypothesized that heightened feelings of attachment security would mitigate the cross-race effect by improving the processing of outgroup-race faces, as well as lessen the tendency to categorize Ambiguous faces as their minority race. However, the main model of prime group and racial essentialism impacting the cross-race effect was not significant. Feeling more or less experimentally boosted attachment security did not impact the use of race to make category judgements and did not mitigate the cross-race effect. At the group level, the distances between Black faces were consistently smaller than the distances between both White and Ambiguous faces for the securely and neutrally primed participants. When comparing individual differences in dimension weights, similarity ratings, and variation between those similarity ratings within face types, there were no significant differences between the securely primed and neutrally primed participants. In addition, security priming did not have a significant main effect on the perceptions of race in the 20 face stimuli or on how varied the ratings were within sets of Black, White, and Ambiguous face stimuli. More variation in ratings of Black and White faces would indicate a more nuanced perception of race, whereas using the endpoints of the scale would indicate a categorical and rigid view of race. Higher variation in Ambiguous face ratings was expected, and the relevant finding would be participants in one group tending to rate Ambiguous faces as more Black overall, but this trend of hypodescent of Ambiguous faces was not observed when averaging between prime conditions. Given the lack of significant findings for heightened security mitigating racial perceptual biases, perhaps in white populations, it may be concluded that heightened security does not impact implicit or explicit perceptions of race and is limited to attitudinal-level judgements. Previous ERP studies would suggest that security priming would influence the emotional processing of face and threat stimuli (Bai et al., 2019; Dickter \& Bartholow, 2007), but this effect on processing did not translate to the current study which used non- 
threatening and non-attachment related faces to assess visual processing and categorization biases. An important note is that the effect of the implicit security prime was not directly tested with a manipulation check, so it is also possible that the security priming effect was smaller in the second session compared to the first, resulting in null findings.

With the main study hypothesis disconfirmed, other potential moderators were considered that could have been masking the effects of the security prime. Because the cross-race effect has been effectively moderated by ingroup identification in past studies (e.g., Hehman, Mania, \& Gaertner, 2010), I examined participant ethnicity which would likely influence ingroup perceptions, as well as participant's reported identification with white identity, where the strength of identification may determine the level of ingroup-face processing bias because of how ingroup reinforcement strengthens holistic processing of ingroup faces (Hugenberg \& Cornielle, 2009). These supplementary analyses illuminated the role of white ingroup identification on perceiving the similarity between same-race faces, but more importantly the moderating role the security prime played in this effect. After excluding Black participants, higher white ingroup identification interacted with the security prime to increase the outgroup homogeneity bias in Ambiguous faces where they were rated as more similar on average. This effect was coupled with higher white ingroup identification leading to lower variation in the similarity ratings across all the Ambiguous face pairs, but this effect was not moderated by the security prime. The simple slopes depicted in Figure 5 highlight the trend that for those with lower white ingroup identification, the security prime impacted the cross-race effect as originally hypothesized, with lower ratings of similarity and a larger variation in similarity ratings indicating better individuation; however, as white ingroup identification increased, the security prime was counterproductive in reducing the cross-race effect, instead enhancing the tendency to judge all Black faces as appearing more similar. In general, higher white ingroup identification was associated with higher similarity ratings of same-race faces, potentially indicating that those individuals preferred to use group membership rather than individual characteristics to assess the similarity of faces, but especially when they were securely primed. 
These findings are not consistent with how activation of a secure attachment system has been understood to promote cognitive openness and exploration (Gillath et al., 2016). Instead, enhanced feelings of security functioned to further situate outgroup homogeneity bias at the perceptual level for highly white identifying participants. A supplementary analysis including the Felt Security measure taken during Session 1 revealed that felt security did mediate at least one facet of perceptual bias (perceived similarity of Ambiguous stimuli), suggesting that it was through heightened feelings of security that white ingroup identification increased the perceived similarity of same-race faces. It is plausible that heightened feelings of security served to empower white individuals' tendency to use group membership in their similarity judgements, in which case the current understanding of the impact of attachment security priming would need to be revised. Security priming has generally been assumed to enhance prosocial attitudes due to its mechanism of facilitating feelings of security and improving cognitive openness (Mikulincer \& Shaver, 2001), but in the context of implicit race cognition and categorization biases, security priming augments participants' established motivations whether or not they are prosocial.

A different parallel mechanism to explain this could be mortality salience priming, which had previously been discussed as a counterpart to security priming where upon priming death-related thoughts, individuals are motivated to reduce that anxiety by situating in their worldviews (Greenberg, Pyszczynski \& Solomon 1986). Mortality salience priming often motivates individuals to become more conservative, self-preservatory, and increase their self-esteem through validating worldviews, oftentimes leading to increased intergroup bias (Castano et al., 2002). One possible explanation for the unexpected effect of the current study's security prime is that the nature of the repeated prime did not work as intended: participants were tasked with thinking about a time when they felt distress and to remember their attachment figure comforting them at that time, then were subliminally shown attachment-related words. The priming manipulation check in the first session confirmed that the participants who received the explicit security prime felt significantly more secure than the neutral participants, but it is possible that security was not the feeling primed through the implicit priming activity, which was not tested with a manipulation check. In studies where attachment and death-thought accessibility were tested together, 
participants who thought about life or relationship disruptions showed higher death-thought accessibility (Yaakobi, Mikulincer \& Shaver, 2014), one of the precursors to intergroup bias according to terrormanagement theory. If the attachment system was functioning in more of a 'tripartite security system' with self-esteem and worldview as discussed in Hart, Shaver, and Goldenberg (2005), then it is plausible that at some point during the current study (e.g., when asked to recall a time of distress or during the implicit prime), participants in the secure group experienced a threat to their attachment system that bolstered defensive mechanisms about worldviews in addition to heightened security. Priming mortality salience has been associated with higher identification with ones' ingroup as well as a heightened sense of the ingroup as an entity (Castano et al., 2002), which could explain why those with higher white ingroup identification tended to rely on group membership for similarity judgements. Studies in which an attachment security prime was used in addition to a mortality salience prime showed that the security prime mitigated the death-thought accessibility from the mortality salience prime (Cox et al., 2008), but also that this attenuation was more effective when the prime was consistent with participants' "core strivings" (i.e., Yaakobi, Mikulincer \& Shaver, 2014). Relatedly, low white-identifying participants who were securely primed tended to individuate same-race faces more, while high white-identifying participants relied on racial group membership for categorization.

In contrast, white ingroup identification did not have an impact on the perceived race of the 20 face stimuli. Rather, there was a main effect of the prime on the perceived race ratings as well as the variability in Ambiguous face ratings, but only in non-white participants. The subsample sizes in these analyses were quite small, with just 16 non-white participants in the secure group and 18 in the neutral group. Nevertheless, the pervasiveness of the prime effect on exclusively non-white participants across several variables merits further discussion. The ethnicity of participants interacted with the security prime such that for non-white individuals, higher security lowered the use of all dimensions in the 4dimensional solution. For these securely primed, non-white participants, there was likely a different dimensional representation that fit their ratings better, or perhaps a fifth dimension would have captured the variance lost in the first four. Importantly, the reliance on the race dimension was significantly 
diminished in non-white participants who were securely primed; in contrast, the average importance of the race dimension for neutrally primed non-white participants was higher than that of the white participants. This suggests that for non-white participants, but not white participants, the security prime can work to reduce the reliance on race to make category judgements.

Ethnicity also interacted with the prime effect on perceived race ratings, where non-white participants displayed more categorical ratings for Black faces after being securely primed. The security prime did not impact white participant ratings of the perceived race of Black faces. Given that the secure prime may have reinforced ingroup identity, as seen by its interaction with white ingroup identity and similarity ratings, this finding is similar to Gaither and colleagues' (2016) speculation that reinforcement of ingroup identity compelled Black participants in their study to include Ambiguous faces into their ingroup in a Black-White rating paradigm. In this case, it appears that the non-white participants (mostly driven by Hispanic/LatinX and Black subgroups) preferred to rate all Black faces as categorically Black rather than offer variation in ratings on the continuous scale, controlling for racial essentialism. Moreover, ethnicity again interacted with the security prime in affecting the variation of Ambiguous face ratings on the perceived race scale. In this case, non-white participants who were securely primed showed a much larger variation in ratings of Ambiguous faces, meaning these participants more often rated Ambiguous faces more extremely Black or White rather than defaulting to the middle rating of "Multiracial." It is unclear what this finding represents in terms of hypodescent and racial essentialism, but it does imply that these participants were more conscious of the "Blackness" versus "whiteness" of these Ambiguous faces and utilized the range of the scale more than other participants. Perhaps the non-white participants felt more empowered due to the heightened security to make ingroup/outgroup judgements about the ambiguous face stimuli. It is possible that a security prime induces more confidence, as that is the basis from which we feel comfortable exploring our environment when feeling securely attached, as discussed earlier with the secure prime enhancing white ingroup members' tendency to use group membership to make similarity judgements. 
Given the small subsample sizes of the interactions involving white/non-white participants, it cannot be said with confidence that these findings will generalize to a larger population. However, considering the congruency in the trends that the securely primed non-white participants had 1) lower reliance on all dimension weights, 2) rated Black faces closest to the category endpoint, and 3) varied largely in their ratings of Ambiguous faces, it is worth investigating further the possible moderation of a security prime with non-white individuals.

\section{Limitations}

One main limitation of this study was the failure to recruit a more diverse sample, though the sample collected was representative of the student population at Illinois State University as of 2019 (71\% white, $11 \%$ Hispanic or Latino, 9\% Black or African American, 3\% Asian; IPEDS, 2019). Cross-race effect research is saturated with majority white university samples, and there is a detrimental lack of documentation of this effect in non-white populations in both Westernized and non-Westernized communities. It is imperative for generalizability that racial-ethnically diverse samples are collected, and this sentiment is further motivated by the findings that ethnicity significantly interacted with how feelings of security impacted perceptions of different race faces. The conception of the attachment security prime as a general tool for improving prosocial relations and lowering intergroup bias cannot be readily supported considering the interaction with ethnicity and white ingroup identification found in this study.

Another limitation was the decision not to include a second priming manipulation check after the implicit priming task. This was done in consideration of the duration of the experiment, as well as of the format of the Felt Security scale which was written to be used after performing an explicit priming task. It was not anticipated that either the explicit or implicit prime would work oppositely as expected in impacting racial essentialism and perceptions of different race faces - taking a short measure of mood and security would have helped to clarify reasons for these differences. In addition, manipulation checks for attention were not implemented throughout the priming and rating tasks, and though there were substantive patterns in the dimensions derived from the nMDS scaling, it is possible these patterns would have been different when only including participants who passed attention checks. Still, one could argue 
that the act of finishing the 40 trial rounds and 200 similarity ratings was a test of attention in of itself, as anyone less committed would likely stop the experiment partway through (as many did).

There was also a novel measure used in this study which was originally conceived to verify that the race dimension appeared in the nMDS solutions, but it was also used as a proximal measure of the cross-race effect which it had not been validated for. Consequently, interpretations regarding the perceived race ratings are limited as it is unconfirmed whether the distribution of ratings in this measure are truly capturing constructs related to the cross-race effect. Additionally, the use of naturally-varying face stimuli was both a limitation and a benefit, as it allowed for increased generalizability of the nMDS solutions, but limited the degree to which we could determine if certain distances between stimuli were objectively true or were reflecting perceptual biases.

This study was also not without technical limitations. Due to the completely online format of this study, it was not possible to maintain the level of experimental control usually achieved for implicit priming paradigms. Participant internet speeds, computer or laptop framerates and processing power could have all affected how the experiment displayed onscreen. A number of participants accurately reported more than three of the five implicit prime words, indicating that there was inconsistency in how long the primes showed onscreen across participants. There was also no guarantee that participants would complete Sessions 1 and 2 in the designated timeframes. Indeed, there were several participants who took time between the first and second tasks in Session 2 that could not be controlled, potentially mitigating the efficacy of the prime. An implicit security priming study which also implements a check for the priming effect in terms of security and other dimensions, such as mood and anxiety, would be a beneficial follow-up to the findings from this study. There was also the unfortunate failure of Qualtrics to counterbalance the perceived race scale as originally intended, so all participants saw the version of the scale where 0 corresponded to Black and 100 to White. Nonetheless, the online format substantially increased the accessibility of the experiment. There was an attrition rate of nearly two-thirds across sessions 1 and 2, but because of the advertisement through mass email, the study easily achieved participation past the target size despite being a multipart, intensive study. 


\section{Future Directions}

The repeated secure attachment prime procedure impacted participants differently than previous literature would suggest, and it may have been due to the online and self-guided administration of both primes, as well as due to interactions with participant ethnicity. A future study could investigate this interaction while making small improvements to the design, such as using manipulation checks after each prime that test for more than just feelings of security, specifically recruiting non-white participants and achieving a more racial-ethnically balanced sample, and by using a scaling procedure that is less prone to respondent fatigue, such as arranging the faces in a plane in whatever clusters and distances are most intuitive. Additionally, it is important to study how the cross-race effect at the perceptual level contributes to more general cognitive-attitudinal biases, such as the outgroup homogeneity bias and group attribution error, and whether this relation would be moderated by a security prime because of its established attitudinal impacts.

It would also be valuable for additional research to investigate the interaction of the attachment security prime with ingroup identities, and to determine whether the interaction from this study is specific to racial ingroup identification or if it can extend further. This can be accomplished by making salient different types of social ingroups the participants may belong to and testing whether their identification with those ingroups impacts similarity ratings, then if these ratings are moderated by a security prime. Subsequently, one should test whether the moderating effect of the security prime feeds into behavioral domains such as attribution error and attitudes towards transgressions made by an outgroup member.

Additional research is needed to clarify how racial essentialism impacts the perceptions of faces when not using a simple Black-White categorizing paradigm, because racial essentialism was not significantly predictive of rating Ambiguous faces as Black when participants were given a full range of options. A future study could have participants choose from two options to describe the face stimuli, one option presenting three simple category checkboxes (Black, Multiracial, White), and the other option presented as a continuous or interval scale similar to the one used in this study. Consolidating the findings from this study with the construct of racial essentialism as the rigid conception of race, it could be 
hypothesized that higher racial essentialism would predict the use of the categorical measure more often than the nuanced measure. Racial essentialism was also not found to be a significant predictor of the implicit perceptual structures participants used in this study, but further research into what other aspects of cognitive-perceptual biases racial essentialism contributes to are necessary to corroborate the findings from this study. It is possible racial essentialism only impacts perceptions when there are explicit task goals about race, and a future study could compare the effects of racial essentialism when participants are given a face sorting task with and without the explicit mention of race.

\section{Conclusions}

The primary goal of this study was to investigate how feelings of security would impact the way ingroup, outgroup, and Ambiguous faces were perceived, and to identify whether security priming could be a useful intervention to mitigate the cross-race effect and its detrimental impact on eye-witness testimonies and more general contribution to outgroup homogeneity biases and attribution errors. It was the first to investigate the perceptual impacts of a security prime using multidimensional scaling and provided insight into the bridge between the cross-race effect, ingroup face perception, and attachment security. The findings indicated that security priming did not have a main effect on the perceptual structures participants used to make category judgements, or on whether they perceived Ambiguous faces as Black or White. Additionally, racial essentialism did not moderate the effect of the security prime for the implicit perceptual structures. Racial essentialism also did not predict hypodescent of ambiguous-race faces when given the ability to make nuanced racial judgements, but instead predicted the use of racial group membership rather than more individuation when perceiving the race of Black face stimuli.

It was suggested from supplementary analyses that the security prime functioned to moderate the impact of white ingroup identification on the perceived similarity between Ambiguous faces, but, unexpectedly, the effect served to heighten the perceptual homogeneity bias for highly white-identifying participants instead of mitigating it. Additionally, there was a significant interaction of the priming effect with participant ethnicity, though this effect requires larger recruitment of non-white individuals to confirm. 
In this sample of mainly white university students, race was the main dimension by which Black, Ambiguous, and White face stimuli were perceived and compared, even when given absolute freedom in the method by which to compare them. Countering the statement that one can be "color-blind," this finding shows that we do see race, and we see it at the level of early perception that we likely are not entirely in control of. 


\section{REFERENCES}

Bai, X., Chen, X., Zhou, M., Liu, C., \& Hu, Y. (2019). The effects of negative context and attachment security priming on working memory updating among anxiously attached individuals. Biological psychology, 143, 41-52. https://doi.org/10.1016/j.biopsycho.2019.02.006

Banks, R. R., \& Eberhardt, J. L. (1998). Social psychological processes and the legal bases of racial categorization. In J. L. Eberhardt \& S. T. Fiske (Eds.), Confronting Racism: The Problem and the Response (pp. 54-75). Sage Publications, Inc.

Bargh, J. A., \& Chartrand, T. L. (2014). The mind in the middle: A practical guide to priming and automaticity research. In H. T. Reis \& C. M. Judd (Eds.), Handbook of Research Methods in Social and Personality Psychology (p. 311-344). Cambridge University Press.

Boccato, G., Capozza, D., Trifiletti, E., \& Di Bernardo, G. A. (2015). Attachment security and intergroup contact. Journal of Applied Social Psychology, 45(11), 629-647. https://doi.org/10.1111/jasp.12325

Borg, I., \& Groenen, P. J. (2005). Modern multidimensional scaling: Theory and applications. Springer Science \& Business Media. E-book preview accessed via Google Scholar.

Canterberry, M., \& Gillath, O. (2013). Neural evidence for a multifaceted model of attachment security. International Journal of Psychophysiology, 88(3), 232-240. doi:10.1016/j.ijpsycho.2012.08.013

Carnelley, K. B., Bejinaru, M. M., Otway, L., Baldwin, D. S., \& Rowe, A. C. (2018). Effects of repeated attachment security priming in outpatients with primary depressive disorders. Journal of Affective Disorders, 234, 201-206. https://doi.org/10.1016/j.jad.2018.02.040

Carnelley, K. B., Otway, L. J., \& Rowe, A. C. (2015). The effects of attachment priming on depressed and anxious mood. Clinical Psychological Science, 4(3), 433-450. https://doi.org/10.1177/2167702615594998

Cassidy, K. D., Boutsen, L., Humphreys, G. W., \& Quinn, K. A. (2014). Ingroup categorization affects the structural encoding of other-race faces: Evidence from the N170 event-related potential. Social neuroscience, 9(3), 235-248. https://doi.org/10.1080/17470919.2014.884981 
Carroll, J. D., \& Chang, J. J. (1970). Analysis of individual differences in multidimensional scaling via an N-way generalization of "Eckart-Young" decomposition. Psychometrika, 35(3), 283-319. https://doi.org/10.1007/BF02310791

Castano, E., Yzerbyt, V., Bourguignon, D., \& Seron, E. (2002). Who may enter? The impact of ingroup identification on ingroup/outgroup categorization. Journal of Experimental Social Psychology, 38, 315- 322. doi: 10.1006/jesp.2001.1512

Chao, M. M., Hong, Y. Y., \& Chiu, C. Y. (2013). Essentializing race: Its implications on racial categorization. Journal of Personality and Social Psychology, 104(4), 619.

Cox, C. R., Arndt, J., Pyszczynski, T., Greenberg, J., Abdollahi, A., \& Solomon, S. (2008). Terror management and adults' attachment to their parents: The safe haven remains. Journal of Personality and Social Psychology, 94(4), 696.

Dickter, C. L., \& Bartholow, B. D. (2007). Racial ingroup and outgroup attention biases revealed by event-related brain potentials. Social cognitive and affective neuroscience, 2(3), 189-198. https://doi.org/10.1093/scan/nsm012

Dodson, C. S., \& Dobolyi, D. G. (2016). Confidence and eyewitness identifications: The cross-race effect, decision time and accuracy. Applied Cognitive Psychology, 30(1), 113-125. https://doi.org/10.1002/acp.3178

Donbaek, F. D., \& Elklit, A. (2014). A validation of the Experiences in Close Relationships-Relationship Structures scale (ECR-RS) in adolescents. Attachment \& Human Development, 16(1), 58-76.

Donovan, B. M. (2014). Playing with fire? The impact of the hidden curriculum in school genetics on essentialist conceptions of race. Journal of Research in Science Teaching, 51(4), 462-496.

Dunham, Y., Stepanova, E. V., Dotsch, R., \& Todorov, A. (2015). The development of race-based perceptual categorization: Skin color dominates early category judgments. Developmental Science, 18(3), 469-483. https://doi.org/10.1111/desc.12228 
Estudillo, A. J., Lee, J. K. W., Mennie, N., \& Burns, E. (2020). No evidence of other-race effect for Chinese faces in Malaysian non-Chinese population. Applied Cognitive Psychology, 34(1), 270276.

Fitzgerald, L. F., \& Hesson-McInnis, M. (1989). The dimensions of sexual harassment: A structural analysis. Journal of Vocational Behavior, 35(3), 309-326.

Fraley, R. C., Heffernan, M. E., Vicary, A. M., \& Brumbaugh, C. C. (2011). The Experiences in Close Relationships-Relationship Structures questionnaire: A method for assessing attachment orientations across relationships. Psychological Assessment, 23, 615-625.

Freiburger, T. L., \& Sheeran, A. M. (2020). The joint effects of race, ethnicity, gender, and age on the incarceration and sentence length decisions. Race and Justice, 10(2), 203-222.

Henry, E.A., Bartholow, B.D., \& Arndt, J. (2010). Death on the brain: Effects of mortality salience on the neural correlates of ingroup and outgroup categorization. Social cognitive and affective neuroscience, 5(1), 77-87. https://doi.org/10.1093/scan/nsp041

Hout, M. C., Papesh, M. H., Goldinger, S. D. (2013). Multidimensional scaling. WIREs Cogn Sci, 4, 93103. doi: $10.1002 /$ wcs. 1203

Gaither, S. E., Pauker, K., Slepian, M. L., \& Sommers, S. R. (2016). Social belonging motivates categorization of racially ambiguous faces. Social cognition, 34(2), 97-118.

Gaither, S. E., Schultz, J. R., Pauker, K., Sommers, S. R., Maddox, K. B., \& Ambady, N. (2014). Essentialist thinking predicts decrements in children's memory for racially ambiguous faces. Developmental Psychology, 50(2), 482.

Gillath, O., Karantzas, G. C., Fraley, R. C. (2016). What can social cognition and priming tell us about attachment? In Adult Attachment: A Concise Introduction to Theory and Research (pp. 159-168). Elsevier.

Greenberg, J., Pyszczynski, T., \& Solomon, S. (1986). The causes and consequences of a need for selfesteem: A terror management theory. In Public Self and Private Self(pp. 189-212). Springer, New York, NY. 
Hehman, E., Mania, E. W., \& Gaertner, S. L. (2010). Where the division lies: Common ingroup identity moderates the cross-race facial-recognition effect. Journal of Experimental Social Psychology, $46(2), 445-448$.

Ho, A. K., Roberts, S. O., \& Gelman, S. A. (2015). Essentialism and racial bias jointly contribute to the categorization of multiracial individuals. Psychological Science, 26(10), 1639-1645. https://doiorg.libproxy.lib.ilstu.edu/10.1177/0956797615596436

Hugenberg, K., \& Corneille, O. (2009). Holistic processing is tuned for in-group faces. Cognitive Science, 33, 1173-1181. doi:101111/j.1551-6709.2009.01048.x

Hugenberg K., Miller J., Claypool H. M. (2007). Categorization and individuation in the cross-race recognition deficit: Toward a solution to an insidious problem. Journal of Experimental Social Psychology, 43, 334-340.

Knowles, M. L., \& Gardner, W. L. (2008). Benefits of group membership: The activation and amplification of group identities in response to social rejection. Personality and Social Psychology Bulletin, 34, 1200-1213. doi: 10.1177/0146167208320062

Klahr, D. (1969). A monte carlo investigation of the statistical significance of Kruskal's nonmetric scaling procedure. Psychometrika, 34(3), 319-330. https://doi.org/10.1007/BF02289360

Luke, M. A., Sedikides, C., \& Carnelley, K. (2012). Your love lifts me higher! The energizing quality of secure relationships. Personality and Social Psychology Bulletin, 38(6), 721-733. https://doi.org/10.1177/0146167211436117

Marsh, B. U., Pezdek, K., \& Ozery, D. H. (2016). The cross-race effect in face recognition memory by bicultural individuals. Acta psychologica, 169, 38-44.

Mikulincer, M., \& Arad, D. (1999). Attachment working models and cognitive openness in close relationships: A test of chronic and temporary accessibility effects. Journal of personality and social psychology, 77(4), 710. https://doi.org/10.1037/0022-3514.77.4.710 
Mikulincer, M., \& Shaver, P. R. (2001). Attachment theory and intergroup bias: Evidence that priming the secure base schema attenuates negative reactions to out-groups. Journal of personality and social psychology, 81(1), 97. https://doi.org/10.1037/0022-3514.81.1.97

Mikulincer, M., Shaver, P. R., \& Rom, E. (2011). The effects of implicit and explicit security priming on creative problem solving. Cognition and Emotion, 25(3), 519-531.

doi:10.1080/02699931.2010.540110

Mikulincer, M., Sheffi, E. (2000). Adult attachment style and cognitive reactions to positive affect: A test of mental categorization and creative problem solving. Motivation and Emotion, 24, 149-174. https://doi.org/10.1023/A:1005606611412

Mondloch, C. J., Elms, N., Maurer, D., Rhodes, G., Hayward, W. G., Tanaka, J. W., \& Zhou, G. (2010). Processes underlying the cross-race effect: An investigation of holistic, featural, and relational processing of own-race versus other-race faces. Perception, 39(8), 1065-1085. doi:10.1068/p6608

Musil, C. M., Warner, C. B., Yobas, P. K., \& Jones, S. L. (2002). A comparison of imputation techniques for handling missing data. Western Journal of Nursing Research, 24(7), 815-829. https://doi.org/10.1177/019394502762477004

Papesh, M. H., \& Goldinger, S. D. (2010). A multidimensional scaling analysis of own-and cross-race face spaces. Cognition, 116(2), 283-288.

Pauker, K., \& Ambady, N. (2009). Multiracial faces: How categorization affects memory at the boundaries of race. Journal of Social Issues, 65(1), 69-86.

Pauker, K., Carpinella, C., Meyers, C., Young, D. M., \& Sanchez, D. T. (2018). The role of diversity exposure in Whites' reduction in race essentialism over time. Social Psychological and Personality Science, 9(8), 944-952.

Pauker, K., Weisbuch, M., Ambady, N., Sommers, S. R., Adams, R. B., \& Ivcevic, Z. (2009). Not so black and white: memory for ambiguous group members. Journal of Personality and Social Psychology, 96(4), 795-810. https://doi.org/10.1037/a0013265 
Rowe, A. C., Gold, E. R., \& Carnelley, K. B. (2020). The effectiveness of attachment security priming in improving positive affect and reducing negative affect: A systematic review. International Journal of Environmental Research and Public Health, 17(3), 968.

Saleem, M., Prot, S., Cikara, M., Lam, B. C., Anderson, C. A., \& Jelic, M. (2015). Cutting Gordian knots: Reducing prejudice through attachment security. Personality and Social Psychology Bulletin, 41(11), 1560-1574.

Shriver, E. R., Young, S. G., Hugenberg, K., Bernstein, M. J., \& Lanter, J. R. (2008). Class, race, and the face: Social context modulates the cross-race effect in face recognition. Personality and Social Psychology Bulletin, 34(2), 260-274. https://doi.org/10.1177/0146167207310455

Slepian, M. L., Weisbuch, M., Pauker, K., Bastian, B., \& Ambady, N. (2014). Fluid movement and fluid social cognition: Bodily movement influences essentialist thought. Personality and Social Psychology Bulletin, 40(1), 111-120.

Subramanian, R., Riley, C., \& Mai, C. (2018). Divided justice: Trends in Black and white jail incarceration, 1990-2013. New York: Vera Institute of Justice.

Stahl, J., Wiese, H., Schweinberger, S. R. (2008). Expertise and own-race bias in face processing: An event-related potential study. Neuroreport, 19, 583-587. doi:10.1097/WNR.0b013e3282f97b4d

Stoet, G. (2010). PsyToolkit - A software package for programming psychological experiments using Linux. Behavior Research Methods, 42(4), 1096-1104.

Stoet, G. (2017). PsyToolkit: A novel web-based method for running online questionnaires and reactiontime experiments. Teaching of Psychology, 44(1), 24-31.

Stolier, R., Freeman, J. (2016). Neural pattern similarity reveals the inherent intersection of social categories. Nat Neurosci, 19, 795-797. https://doi.org/10.1038/nn.4296

Tangen, J. M., Murphy, S. C., \& Thompson, M. B. (2011). Flashed face distortion effect: Grotesque faces from relative spaces. Perception, 40(5), 628-630. 
Tham, D. S. Y., Bremner, J. G., \& Hay, D. (2017). The other-race effect in children from a multiracial population: a cross-cultural comparison. Journal of Experimental Child Psychology, 155, 128137.

Tropp, L. R., \& Wright, S. C. (2001). Ingroup identification as the inclusion of ingroup in the self. Personality and Social Psychology Bulletin, 27(5), 585-600. https://doi.org/10.1177/0146167201275007

Williams, M. J., \& Eberhardt, J. L. (2008). Biological conceptions of race and the motivation to cross racial boundaries. Journal of Personality and Social Psychology, 94, 1033-1047. https://doi.org/10.1037/0022-3514.94.6.1033

Yaakobi, E., Mikulincer, M., \& Shaver, P. R. (2014). Parenthood as a terror management mechanism: The moderating role of attachment orientations. Personality and Social Psychology Bulletin, 40(6), $762-774$.

Zhao, M., Hayward, W. G., \& Bülthoff, I. (2014). Face format at encoding affects the other-race effect in face memory. Journal of Vision, 14(9), 6-6.

Zilber, A., Goldstein, A., \& Mikulincer, M. (2007). Adult attachment orientations and the processing of emotional pictures-ERP correlates. Personality and Individual Differences, 43(7), 1898-1907. 


\section{APPENDIX A: MATERIALS USED}

\section{Figure A1.}

Frequency Distribution of Average Ratings on the Perceived Race Scale

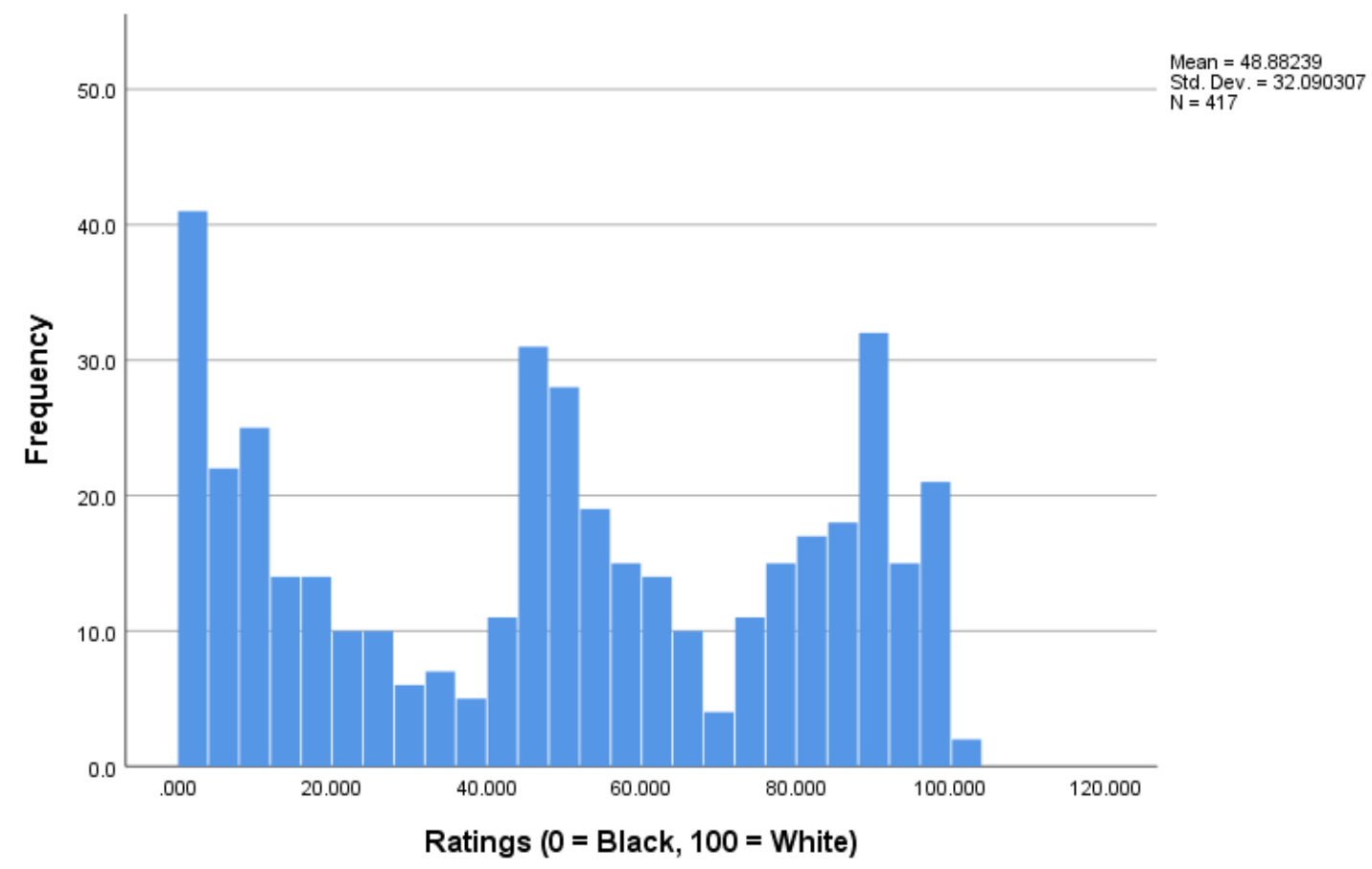

Note. Relatively normal distributions around the middle (Ambiguous) and high (White) end of the scale as well as the right-skewed distribution at the low end of the scale (Black) were expected from the majority White population. 
Figure A2.

Ingroup Identification Scale
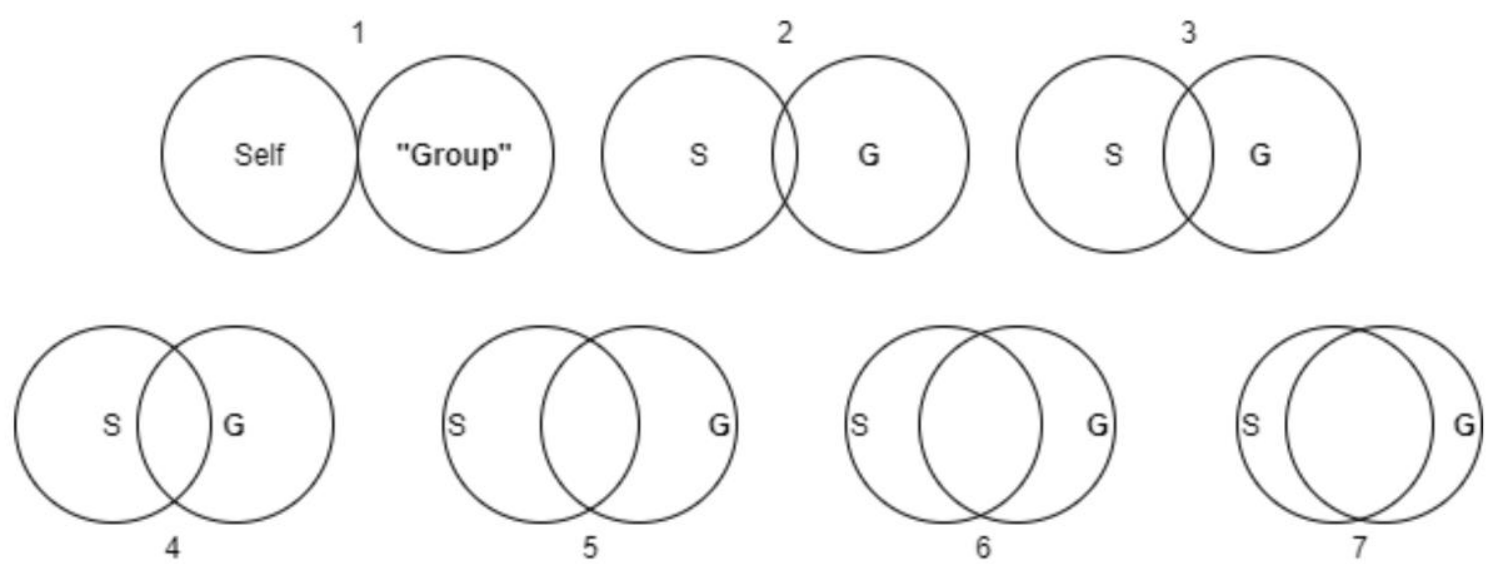

Note. Graphic accompanied by the text: "Given the above diagram, and that 'Group' refers to white [or Black] identity, select the number corresponding to the pair of circles that you feel best represents your own level of identification with the group." 


\section{APPENDIX B: 2D REPRESENTATIONS OF DIMENSIONS}

\section{Figure B1.}

Reduced Multidimensional Representation of Dimension 1 and 2 from the 4-Dimensional Solution

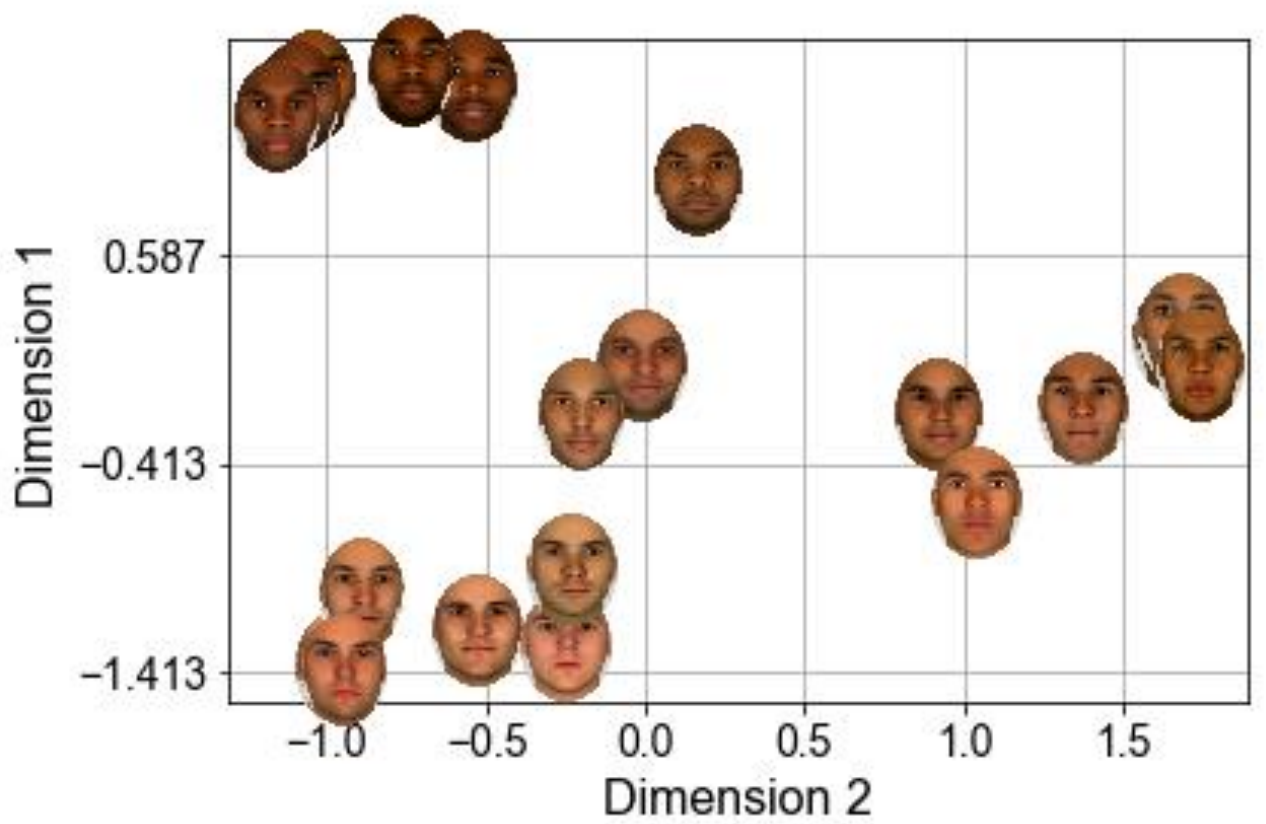

Note. Dimension 2 separates perceived ambiguous faces (right) from the Black and White faces (left).

Notably, two Ambiguous faces load similarly to the other non-ambiguous faces suggesting that they were perceived as either Black or White. 


\section{Figure B2.}

Reduced Multidimensional Representation of Dimension 1 and 3 from the 4-Dimensional Solution

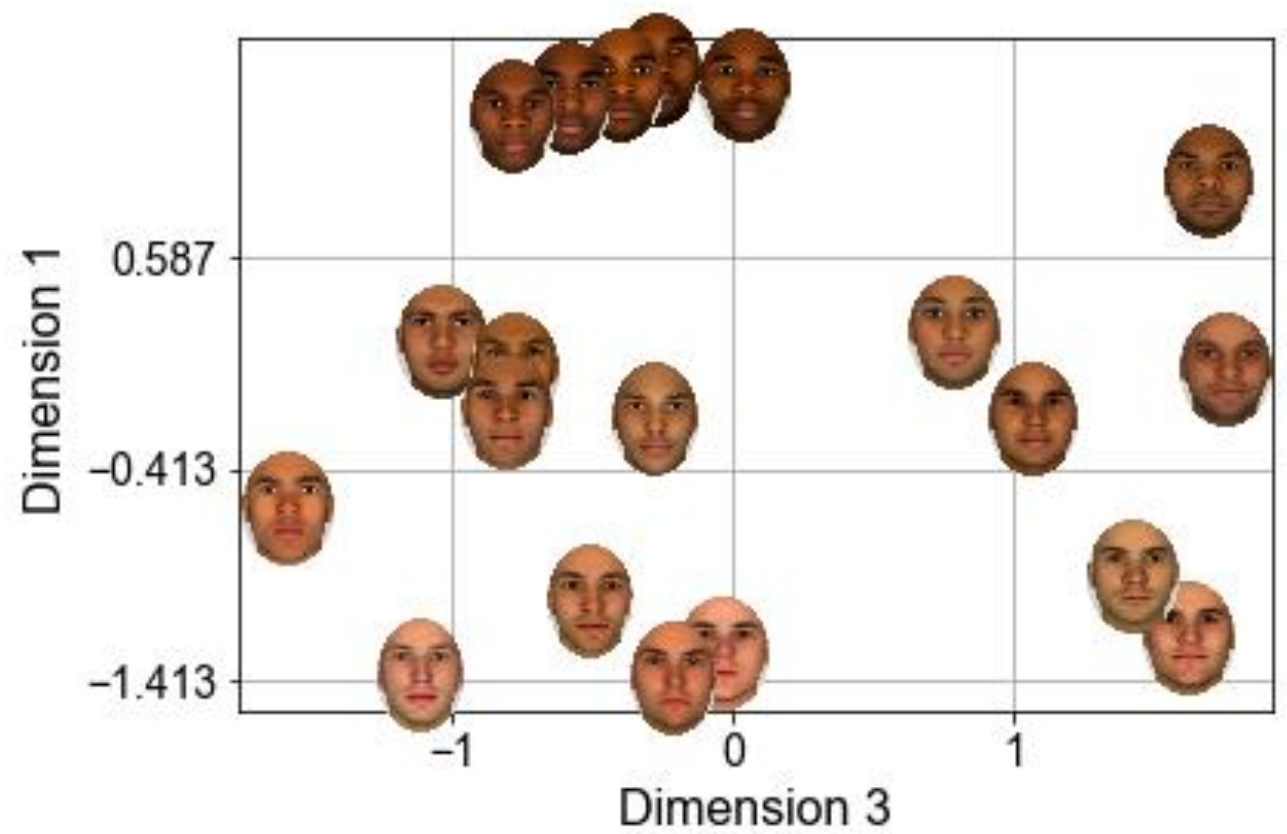

Note. Dimension 3 delineates prototypical (left) from non-prototypical faces (right). The non-prototypical faces each have unique facial characteristics (e.g., skin tone, face structure) that were not similar to any other face within their race type. 


\section{Figure B3.}

Reduced Multidimensional Representation of Dimension 2 and 3 from the 4-Dimensional Solution

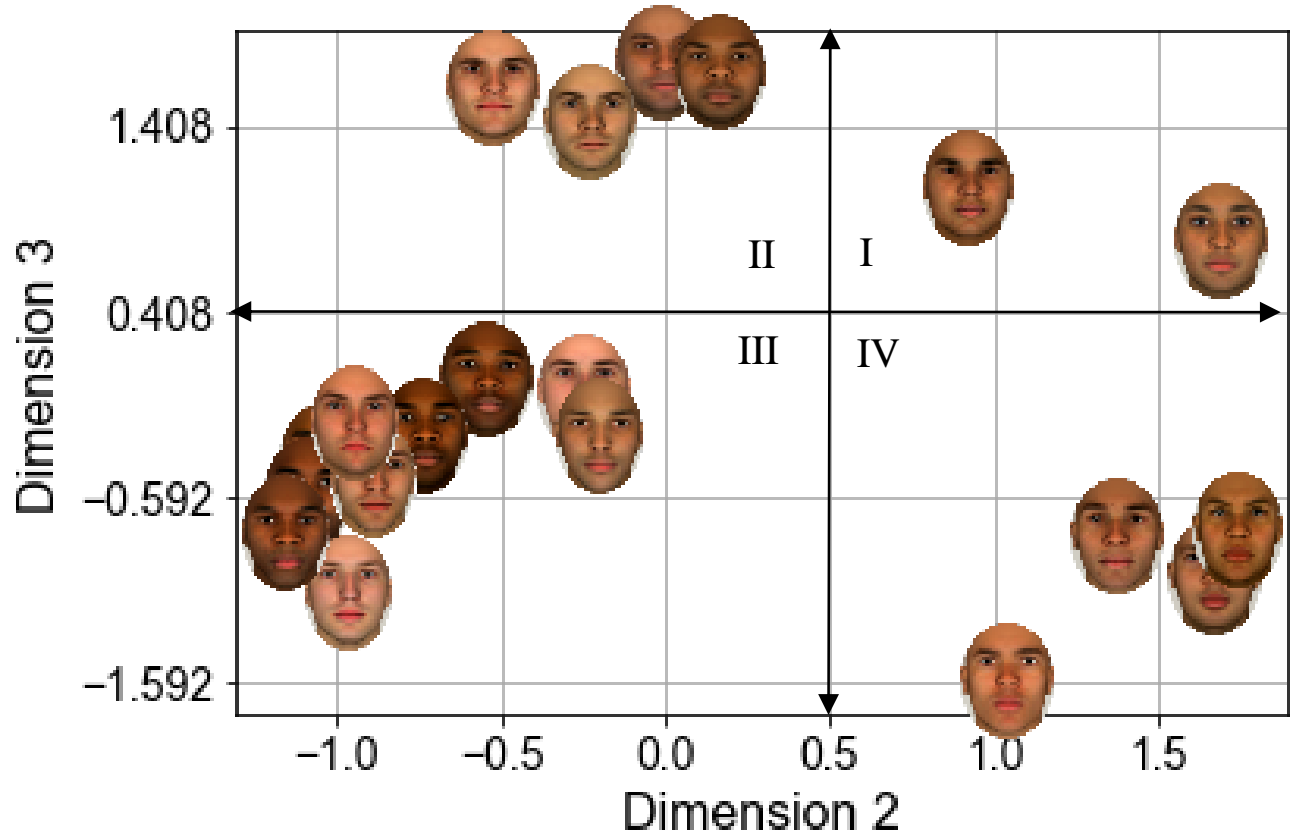

Note. Four quadrants where the left and right quadrants represent non-ambiguous or ambiguous race faces, and the upper and lower quadrants represent non-prototypical or prototypical facial features, respectively. 
Figure B4.

Reduced Multidimensional Representation of Dimension 1 and 4 from the 4-Dimensional Solution

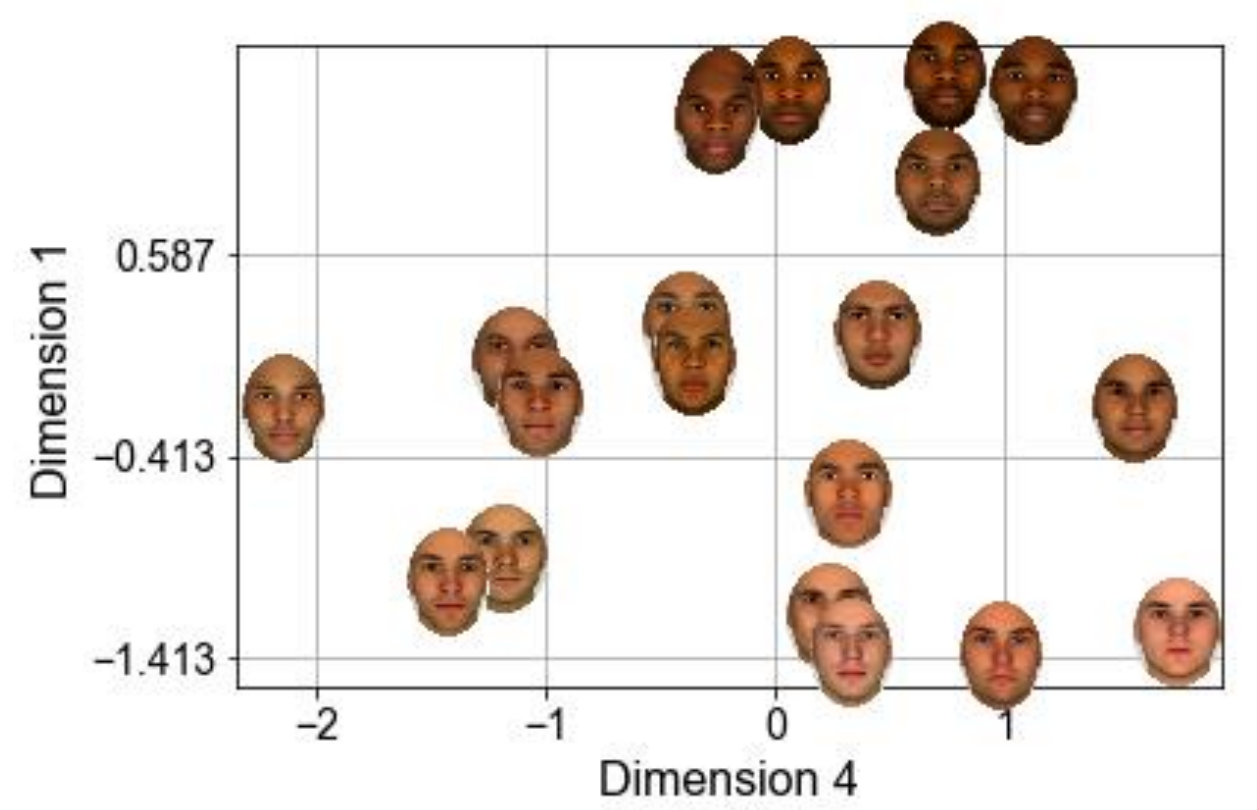

